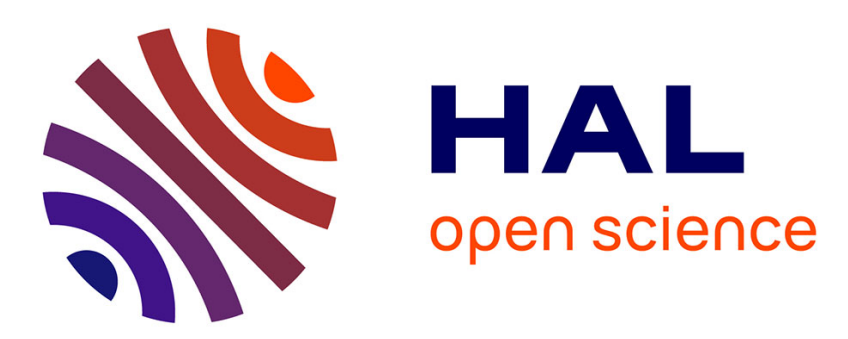

\title{
Large-Eddy Simulations of the Mascotte test cases operating at supercritical pressure
}

Schmitt Thomas

\section{To cite this version:}

Schmitt Thomas. Large-Eddy Simulations of the Mascotte test cases operating at supercritical pressure. Flow, Turbulence and Combustion, 2020, 105 (1), pp.159-189. 10.1007/s10494-019-00096-y . hal-02409214

\section{HAL Id: hal-02409214 \\ https://hal.science/hal-02409214}

Submitted on 10 Dec 2020

HAL is a multi-disciplinary open access archive for the deposit and dissemination of scientific research documents, whether they are published or not. The documents may come from teaching and research institutions in France or abroad, or from public or private research centers.
L'archive ouverte pluridisciplinaire HAL, est destinée au dépôt et à la diffusion de documents scientifiques de niveau recherche, publiés ou non, émanant des établissements d'enseignement et de recherche français ou étrangers, des laboratoires publics ou privés. 


\title{
Large-Eddy Simulations of the Mascotte test cases operating at supercritical pressure
}

\author{
Thomas Schmitt
}

Received: date / Accepted: date

\begin{abstract}
Liquid rocket, Diesel or aircraft engines may operate in the transcritical regime. In such thermodynamic conditions, the classical phase change that occurs at subcritical pressure disappears and the mixing layer between the dense and cold jet and the outer gaseous stream is characterized by large variations of density and thermodynamic properties. Fluids show strong departure from a perfect gas behavior and a real-gas formulation is needed to model the fluid state. The extension of the unstructured AVBP solver, jointly developed by CERFACS and IFPEN, to handle highpressure thermodynamics is presented in details. It is then validated on the experimental coaxial injectors studied with the Mascotte test rig from ONERA that operate in the transcritical range, namely the $\mathrm{LOx} / \mathrm{GH} 2$ cases $\mathrm{A} 60$ and $\mathrm{C} 60$ and the $\mathrm{LOx} / \mathrm{GCH} 4$ configuration G2. The flame pattern observed in experiments is properly recovered, hence validating the numerical strategy. Numerical results are then discussed focusing on the role of the momentum flux ratio on the development of transcritical flames.
\end{abstract}

Keywords Large-Eddy Simulation · transcritical regime · turbulent combustion

\section{Introduction}

Many liquid rocket, Diesel or aircraft engines operate in the transcritical regime. For example, the two $\mathrm{LH}_{2} / \mathrm{LO}_{2}$ liquid rocket engine that equip Ariane 6, Vulcain and Vinci engines, operate at $11 \mathrm{MPa}$ and $6 \mathrm{MPa}$ respectively. This pressure exceeds the critical pressure of oxygen $\left(\mathrm{P}_{C}\left(\mathrm{O}_{2}\right)=5.04 \mathrm{MPa}\right)$, which is otherwise injected at a temperature below its critical temperature $\left(T_{C, O_{2}}=154 \mathrm{~K}\right)$. Under such conditions, the oxygen stream is transcritical and the perfect gas equation of state and associated thermodynamics are not valid anymore [1]. Surface tension and latent heat of vaporization are diminished. As a consequence, atomization and droplet generation processes occuring at subcritical pressure may no longer be present. Instead, the dense oxygen jet diffuses in the surrounding light fluid. This phenomenon has been observed in multiple experiments involving single-species flows, as well as non-reactive and reactive multi-species coaxial injections [2-4]. The development of accurate unsteady reactive simulations under transcritical conditions is then of interest for many applications, especially for rocket applications, and is the subject of this article. It should be pointed out that multi-species mixing may leads to phase separation [5-7]. However, recent studies reveals that it is expected to be of limited importance in the context of reactive flows for rocket engine applications [5,7]. Mixture supercritical conditions are then assumed in this study.

In the reacting cases studied experimentally in [4,8], transcritical oxygen jets are surrounded by high-velocity supercritical hydrogen or methane streams. A pure diffusion flame regime, controlled by turbulent mixing, was observed. Supercritical pressure and the absence of droplets lead to a thin flame located in proximity of the cold and dense oxygen jet. Transcritical oxygen / gaseous

Laboratoire EM2C, CNRS, CentraleSupélec, Université Paris-Saclay,

3, rue Joliot Curie, 91192 Gif-sur-Yvette cedex, France

Tel.: +33175316091

E-mail: thomas.schmitt@centralesupelec.fr, thomas.schmitt@cnrs.fr 
hydrogen or methane flames thus feature a strong turbulent diffusion-flame brush anchored at the injector lip that wraps the central cold oxygen jet.

Numerical simulations show the impact of transcritical conditions on the thermodynamics and flow dynamics [9,10] and combustion [11-15]. Direct Numerical Simulations (DNS) [9, 16] were used to study mixing processes, highlighting the importance of non-ideality in the context of Large-Eddy Simulations (LES) or Reynolds-Averaged-Navier-Stokes simulations (RANS) [17-20]. However it should be noticed that RANS simulations, based on classical closures, were able to provide good representations of the average flame shape [21,22]. Recent efforts focus on the development of LES solvers for transcritical flows to describe the unsteady turbulent flame structure [23-37].

One major issue is the capability of numerical methods to remain stable and accurate in regions of large density gradients. The strong non-linearity of thermodynamic properties renders the system highly sensitive to small variations of density or energy, that may generate high amplitude, unphysical, pressure oscillations $[26,31,38-40]$. These unphysical phenomena, produced in underresolved gradients or during numerical dissipation, may artificially perturb the flow and affect the prediction of combustion instabilities. They should be canceled or, at least, limited. However, classical conservative methods, expected to be prone to undesired pressure fluctuations, have been used with great success by many researchers $[6,10,32,41-44]$. Methods derived to limit or suppress these spurious pressure oscillations are generally non-conservative on energy [26,33,45]. A recent energy conservative strategy for the Van Der Waals equation of state has been proposed by Pantano et al. [46]. In the present work, a non-conservative artificial viscosity is used to stabilize the system $[45]$.

Unsteady numerical studies focused on the vicinity of the coaxial injectors [10,24,47-50], showing a flame anchored in the wake region behind the injector lip and spreading around the LOx jet. Simulations of the whole flame in transcritical regime, from the injection unit to the flame end, have been performed in $[25,27,37,43,51-56]$. Numerical flame structures are generally in reasonable agreement with experimental data when available $[27,53,37]$. However, additional validations of the LES methodology against experimental transcritical flames are still needed.

In the present paper, the unstructured AVBP solver is used to simulate three experimental coaxial flames operating in transcritical conditions. The first objective of this study is to develop and validate a methodology for the AVBP solver to perform Large-Eddy Simulations of reacting flows under transcritical conditions. The numerical strategy, inherited from the method presented in [45], make use of a modified artificial viscosity sensor and is here presented in details. The second goal is to numerically investigate the well-known Mascotte test-cases [4,8]. In contrast to the existing LES of cases C60 and G2 [27,51], the 3 supercritical Mascotte test cases (A60, C60 and G2) are considered within the same framework, allowing for a proper validation of the method and a fair comparison between the cases. Also, in order to limit the CPU cost, the previous calculations were performed using a modified injector geometry, featuring thickened lips [51]. The actual tapered lips are considered in this work. The three cases selected for this study are well suited for code validation as they are known to have three distinct dynamic behaviors [4,8]: a) Case C60 features a long flame, with lot of intermittency in the second part of the chamber ; b) Case A60 is much shorter with a flame almost completely visible in the optical window ; c) Case G2 shows a compact flame, fully visible in the optical window. Finally, the effect of the momentum ratio on the flame development is investigated. This parameter is of major importance in coaxial jet mixing [57] and combustion [8]. Details about the models and the solver are given in Sec. 2. The experimental configurations and the numerical setup are described in Sec. 3. The numerical strategy is then validated in Sec. 4. Results are eventually discussed in Sec. 5, where the complete flame dynamics are detailed. 


\section{Governing equations, models and numerics}

\subsection{Governing equations}

The Favre-filtered, fully compressible Navier-Stokes equations are used to formulate the LargeEddy Simulations (LES) approach [58]:

$$
\begin{aligned}
& \frac{\partial \bar{\rho} \widetilde{Y}_{k}}{\partial t}+\frac{\partial \bar{\rho} \widetilde{Y}_{k} \widetilde{u}_{j}}{\partial x_{j}}=-\frac{\partial \overline{J_{k, j}}}{\partial x_{j}}-\frac{\partial J_{k, j}^{t}}{\partial x_{j}}+\overline{\dot{\omega}_{k}} \\
& \frac{\partial \bar{\rho} \widetilde{u}_{i}}{\partial t}+\frac{\partial \bar{\rho} \widetilde{u}_{i} \widetilde{u}_{j}}{\partial x_{j}}=-\frac{\partial \bar{p}}{\partial x_{i}}+\frac{\partial \overline{\tau_{i, j}}}{\partial x_{j}}+\frac{\partial \tau_{i, j}^{t}}{\partial x_{j}} \\
& \frac{\partial \bar{\rho} \widetilde{E}}{\partial t}+\frac{\partial \bar{\rho} \widetilde{u}_{j} \widetilde{E}}{\partial x_{j}}=-\frac{\partial \bar{p} \widetilde{u}_{j}}{\partial x_{j}}+\frac{\partial \widetilde{u}_{i} \overline{\tau_{i, j}}}{\partial x_{j}}-\frac{\partial \overline{q_{j}}}{\partial x_{j}}-\frac{\partial q_{j}^{t}}{\partial x_{j}}+\overline{\dot{\omega}_{T}}
\end{aligned}
$$

where $\bar{\phi}$ and $\tilde{\phi}$ denote spatial and mass-weighted (Favre) spatial filter, $p$ is the pressure, $T$ the temperature, $\rho$ the density, $Y_{k}$ is the mass fraction of the species $k, u_{i}$ is the velocity vector, $x_{i}$ the three spatial coordinates, $t$ the time, $E$ the total sensible energy, $\tau_{i, j}^{t}$ the sub-grid scale (SGS) stress tensor, $q_{j}^{t}$ the SGS energy fluxes, $J_{k, j}^{t}$ the SGS species fluxes, $\dot{\omega}_{k}$ the species reaction rate and $\dot{\omega}_{T}$ the heat release rate.

The species $\mathbf{J}_{\mathbf{k}}$ and heat fluxes $\mathbf{q}$ use classical gradient approaches. The fluid viscosity and the heat diffusion coefficient are calculated following the Chung et al. method [59] and mass diffusion coefficients are deduced from heat diffusivity by assuming a unity Lewis number (Le=1). The latter assumption is an approximation, especially for $\mathrm{H}_{2} / \mathrm{O}_{2}$ cases $[12,60]$. It is expected not to be so influential for the turbulent cases studied here because the turbulent sub-grid-scale fluxes dominate the laminar fluxes by at least one order of magnitude. Nevertheless, effects of differential diffusion may be significant in the near injector region. Following DNS results of Oefelein [24], the Soret and Dufour effects, which are shown to be much smaller than ordinary diffusion, are neglected.

\subsection{Sub-grid scale models and assumptions}

The sub-grid scale (SGS) energy and species fluxes are modeled using the gradient transport assumption, introducing SGS turbulent viscosity $\nu_{t}$, turbulent species diffusion $D_{t}$ and turbulent thermal conductivity coefficients $\lambda^{t}$ :

$$
\begin{aligned}
\tau_{i j}^{t} & =2 \bar{\rho} \nu_{t}\left(\widetilde{S}_{i j}-\frac{1}{3} \delta_{i j} \widetilde{S}_{l l}\right) \text { with } \widetilde{S}_{i j}=\frac{1}{2}\left(\frac{\partial \widetilde{u}_{j}}{\partial x_{i}}+\frac{\partial \widetilde{u}_{i}}{\partial x_{j}}\right)-\frac{1}{3} \frac{\partial \widetilde{u}_{k}}{\partial x_{k}} \delta_{i j} \\
{\overline{J_{i, k}}}^{t} & =-\bar{\rho}\left(D_{t} \frac{\partial \widetilde{Y}_{k}}{\partial x_{i}}\right) \\
\bar{q}_{i}^{t} & =-\lambda_{t} \frac{\partial \widetilde{T}}{\partial x_{i}}+\sum_{k=1}^{N} \bar{J}_{i, k} t \widetilde{h}_{s, k}
\end{aligned}
$$

with:

$$
D_{t}=\frac{\nu^{t}}{S c_{t}} \text { and } \lambda_{t}=\frac{\rho \nu_{t} C_{p}}{P r_{t}}
$$

where $h_{s, k}$ is the partial-mass sensible enthalpy of species $\mathrm{k}$, and turbulent Prandtl $P r_{t}$ and Schmidt $S c_{t}$ numbers are both set to 0.7 . The SGS turbulent viscosity $\nu_{t}$ is modeled with the WALE model [61], well suited for shear flows [45]. In the absence of fully validated models, additional SGS terms, depicted in Taskinoglu et al. [18] and Borghesi and Bellan [62] and that are negligible in ideal cases, are not considered here. Notably, filtered pressure $\bar{p}$ is computed directly from filtered variables: $\bar{p} \approx p\left(\bar{\rho} \tilde{Y}_{k}, \bar{\rho} \tilde{E}\right)$. This is an approximation that has been shown a posteriori to be reasonable at least for small LES filters [62] but need further investigations for large filters and high Reynolds flows. 


\subsection{Thermodynamics}

Thermodynamic non-idealities are accounted for using the Soave-Redlich-Kwong equation of state [63]:

$$
p=\frac{\rho r T}{1-\rho b_{m}}-\frac{\rho^{2} a_{m}(T)}{1+\rho b_{m}}
$$

where $r=R / \bar{W}$, with $R$ the perfect gas constant and $\bar{W}$ the mean molar mass of the mixture. A classical Van Der Walls mixing rule is used for the computation of the equation coefficients $a_{m}$ and $b_{m}$ (see [1] for details).

All the thermodynamic coefficients are then consistently derived from the equation of state as described in $[1,9,24]$. Under the current simulation thermodynamic, the SRK equation of state accurately predicts density and energy at the injection temperature, and shows a maximum relative error of $12 \%$ and $20 \%$ for oxygen and methane densities near the critical temperature compared with the NIST database [64] (Fig. 1). Possibility of phase separation is neglected in this work. In particular, for the case of $\mathrm{O}_{2} / \mathrm{H}_{2}$ combustion at 60 bar, it has been shown that liquid water may appears near the cold oxygen jet [5] but in small quantities, because the species mixing essentially occurs in the high temperature region of flame. It is thus considered to have a negligible impact on the flame dynamics and is neglected.
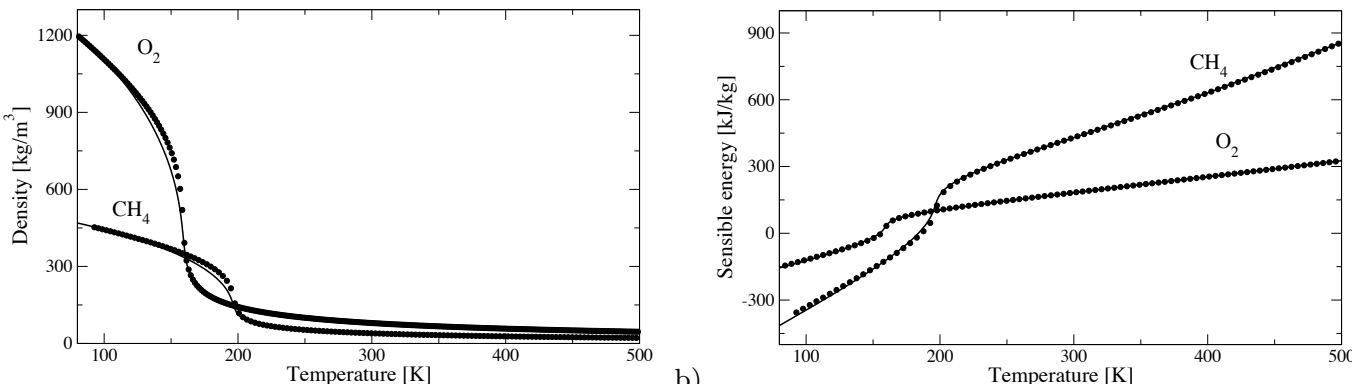

Fig. 1 Comparison of SRK with the NIST database. (a) Density and (b) sensible energy for oxygen at 6.0 MPa and methane at 5.5 MPa computed with the SRK equation of state (continuous line) and from the NIST database (dots) [64].

\subsection{Combustion model}

In high pressure cryogenic rocket engines, combustion operates in a pure diffusion regime $[8,65]$. In addition, since pure reactants are considered in a high pressure environment, a fast chemistry assumption is justified for the cases simulated here $[11,66]$ so that species equilibrium is assumed in this work. In particular, results of $\mathrm{CH}_{4} / \mathrm{O}_{2}$ strained diffusion flame calculations at high pressure [11] indicate that the fast chemistry assumption is accurate for strain rates lower than $9.10^{5} \mathrm{~s}^{-1}$ at 50 bar, whereas the strain rates in the present high-pressure simulation remain essentially lower than $1.10^{5} \mathrm{~s}^{-1}$, justifying the fast chemistry assumption. Moreover, recent two-dimensional highly resolved numerical simulations with complex chemistry and accounting for conjugate heat transfert in the lip region of a $\mathrm{LO}_{2} / \mathrm{GH}_{2}$ flame $[67,68]$ indicate that chemistry is fast. Each species are then deduced from equilibrium calculations as a function of the mixture fraction $Z$. In order to properly represent the temperature over the whole mixture fraction domain, four species $\left(\mathrm{H}_{2} \mathrm{O}, \mathrm{H}_{2}, \mathrm{O}_{2}\right.$ and $\mathrm{OH}$, Fig. 2a) are considered here for cases involving hydrogen - oxygen combustion (Sec. 3.1) and seven species $\left(\mathrm{CH}_{4}, \mathrm{O}_{2}, \mathrm{H}_{2} \mathrm{O}, \mathrm{CO}_{2}, \mathrm{CO}, \mathrm{OH}\right.$ and $\mathrm{H}_{2}$, Fig. 2b) are used for the case that considers methane - oxygen combustion. Main species mass fractions are properly represented. In particular, the shape of $\mathrm{OH}$ mass fraction are recovered for both equilibrium, even though their maximum are overestimated with the simplified equilibrium. The maximum error on temperature is $150 \mathrm{~K}$ for $\mathrm{H}_{2} / \mathrm{O}_{2}(4 \%)$ and $120 \mathrm{~K}$ for $\mathrm{CH}_{4} / \mathrm{O}_{2}(3.5 \%)$, near stoichiometry. Mixture temperature is properly captured for other equivalence ratios. Filtered mass fractions are computed using a $\beta$-pdf $[58,69]$ : 
a)

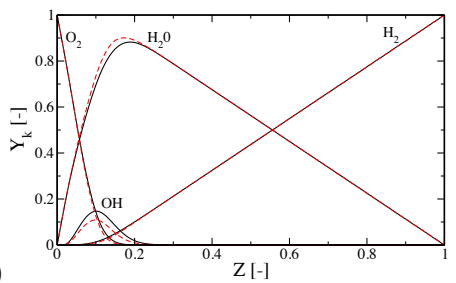

b)

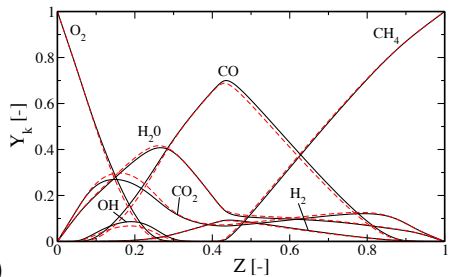

c)

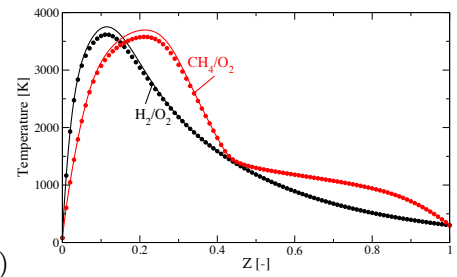

Fig. 2 Equilibrium calculations used in the combustion model. Species distribution in terms of the mixture fraction for (a) cases A60 and C60 and (b) case G2 (Black: equilibrium used in this study, red: complex chemistry equilibirum). (c) Comparison between the temperature predicted from the simplified equilibrium used in this study (continuous line) and complex chemistry (dots).

$$
\widetilde{Y_{k}}\left(\widetilde{Z}, \widetilde{Z^{\prime 2}}\right)=\int_{0}^{1} Y_{k}\left(Z^{*}\right) P\left(Z^{*}, x, t\right) d Z^{*}
$$

where $P$ is the $\beta$-pdf depending on $\widetilde{Z}$ and $\widetilde{Z " 2}$, the filtered variance of the mixture fraction. Both $\widetilde{Z}$ and $\widetilde{Z " 2}$ are transported in the simulation [70]:

$$
\begin{aligned}
\frac{\partial \bar{\rho} \widetilde{Z}}{\partial t}+\nabla \cdot(\bar{\rho} \widetilde{Z} \widetilde{u}) & =\nabla \cdot\left(\bar{\rho}\left(D+D_{t}\right) \nabla \widetilde{Z}\right) \\
\frac{\partial \bar{\rho} \widetilde{Z " 2}}{\partial t}+\nabla \cdot\left(\bar{\rho} \widetilde{Z^{\prime \prime}} \widetilde{u}\right) & =\nabla \cdot\left(\bar{\rho}\left(D+D_{t}\right) \nabla \widetilde{Z^{\prime \prime 2}}\right)+2 \bar{\rho} D_{t}\|\nabla \widetilde{Z}\|^{2}-2 \bar{\rho} D_{t} \frac{\widetilde{Z^{\prime 2}}}{\Delta_{x}{ }^{2}}
\end{aligned}
$$

Finally the strategy employed here allows for an explicit formulation of the filtered reaction rate $\overline{\dot{\omega}_{k}}$ determined from a relaxation between the tabulated filtered mass fraction $\widetilde{Y_{k}}\left(\widetilde{Z}, \widetilde{Z^{\prime \prime 2}}\right)$ and the one transported assuming pure mixing $\widetilde{Y_{k}^{+}}[27,71]$ :

$$
\overline{\omega_{k}}=\bar{\rho} \frac{\widetilde{Y_{k}}\left(\widetilde{Z}, \widetilde{Z^{\prime 2}}\right)-\widetilde{Y_{k}^{+}}}{C_{r} \Delta t}
$$

where $\Delta t$ is the time step and $C_{r}$ a constant that should theoretically be equal to 1 . In practice, it can be set to larger values as long as the resulting characteristic time remains smaller than the other characteristic times of the flow. $C_{r}$ is set to 100 in this paper. The filtered heat release rate is then computed from $\overline{\dot{\omega}_{k}}$ :

$$
\overline{\dot{\omega}_{T}}=-\sum_{k=1}^{N} \Delta h_{f, k}^{0} \overline{\dot{\omega}_{k}}
$$

where $\Delta h_{f, k}^{0}$ is the formation enthalpy of species $k$. Finally, the source terms $\overline{\dot{\omega}_{k}}$ and $\overline{\dot{\omega}_{T}}$ are directly used in Eq. 1 and 3, which allows a simple and direct coupling with the compressible solver.

It should be mentioned that more complex combustion models have been developed for supercritical combustion, relying on complex chemistry and possibly able to account for chemistry effects. These models are based on tabulated chemistry generally using laminar flamelet or flamelet/progress variable approaches [43,72,35,36,30,73,34,37]. Good results have been obtained using flamelet approach in real scale cases $[43,73,37]$ even though further validations are still needed. However, for the configurations under investigation here, a fast chemistry assumption is reasonable.

\subsection{Numerics and stabilization method}

\subsubsection{Description of the method}

The compressible unstructured solver AVBP [74,75] is used for this study. Its Taylor-Galerkin weighted residual central distribution scheme, called TTGC, is third-order in time and space [76]. The Jacobian matrices of non-viscous fluxes used by the scheme are written to be fully consistent with the non-ideal thermodynamics (detailed in Appendix A). Boundaries are treated with 
the characteristic wave decomposition method NSCBC derived for non-ideal thermodynamics as detailed in [77].

Like any non-dissipative centered scheme, TTGC is very sensitive to high gradients and is stabilized applying locally artificial viscosity to guarantee both accuracy and stability. Cells with underresolved wavelength are detected by a density sensor $S_{\rho}$ :

$$
S_{\rho}=\frac{|\boldsymbol{u} \cdot \boldsymbol{n}| \Delta t}{\Delta x}\left|\frac{\widehat{\bar{\rho}}-\bar{\rho}}{0.01 \bar{\rho}}-\xi\right|
$$

where $\Delta t$ is the time step, $\Delta x$ the characteristic cell size, $\boldsymbol{n}$ the normal to the density gradient and $\widehat{\phi}=F * \phi$ denotes a spatial filtering of variable $\phi$. The spatial recursive filter $\mathrm{F}$ used in this work follows the strategy depicted in Mathew and al. [78] based on an extension to the deconvolution method of Stolz et al. [79]. The explicit selective filter $F$ is built from a reference filter $G$ and its approximate deconvolution $Q$ :

$$
\widehat{\bar{\rho}}=F * \bar{\rho}=Q * G * \bar{\rho}=\sum_{n=0}^{N}(I-G)^{n} * G * \bar{\rho}
$$

where $I$ is the identity operator and $G$ is an approximate fourth order derivative [75], its expression at node $i$ is given by:

$$
(G * \bar{\rho})_{i}=\rho_{i}+\frac{1}{V_{i}} \sum_{j \mid i \in \Omega_{j}} \frac{V_{\Omega_{j}}}{n_{v}^{\Omega_{j}}}\left[\left(\frac{1}{n_{v}^{\Omega_{j}}} \sum_{k \in \Omega_{j}} \nabla \bar{\rho}_{k}\right)\left(x_{\Omega_{j}}-x_{i}\right)-\left(\bar{\rho}_{\Omega_{j}}-\bar{\rho}_{i}\right)\right]
$$

where $\phi_{i}$ are the variables taken at the node $i$ and $\phi_{\Omega_{j}}=1 / n_{v}^{\Omega_{j}} \sum_{k \in \Omega_{j}} \phi_{k}$ are the cell averaged variables taken at cell $j, V_{i}$ is the volume at node $i, V_{\Omega_{j}}$ is the volume of cell $j$ and $n_{v}^{\Omega_{j}}$ is the number of vertices of cell $j$. The filter response function for $F$ is plotted in Fig. 3 as a function of the number of recursion. Following the dispersion properties the TTGC scheme (see for example Figs. 6 and 7 of [76]), where dispersion errors are shown to be negligible for normalized wavenumbers lower than 0.3 , only one recursion $(\mathrm{N}=1)$ is used in the sensor. The free parameter $\xi$ acts roughtly as a threshold value and is typically set between $\xi=0.0$ and $\xi=10.0$ in practical simulations.

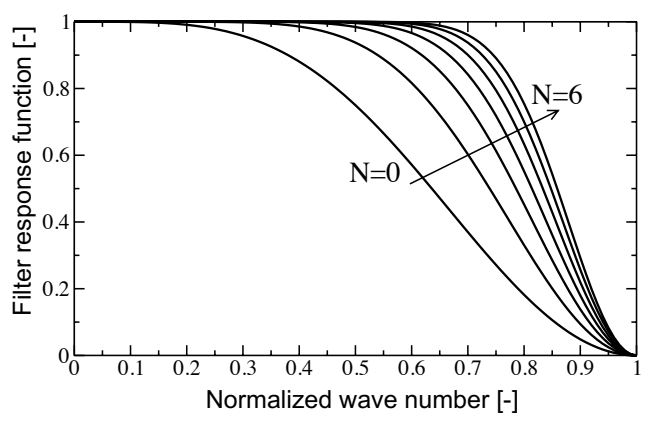

Fig. 3 Filter response function as a function of the wavenumber for different values of the number of recursion $N$. The value $N=1$ is a good candidate for TTG schemes [76] used in the AVBP solver and is chosen in the sensor. $N=0$ actually corresponds to the reference filter $G$, an approximate fourth order derivative.

Finally, limiters for species $S_{Y_{k}}$, mixture fraction $S_{Z}$ and its variance $S_{Z^{\prime \prime}}$ are computed to locate regions with values lower than zero or greater than 1 :

$$
\begin{gathered}
S_{\phi}= \begin{cases}\min \left(\frac{|\phi|}{\epsilon}, 1\right), & \text { if } \phi<0 . \\
\min \left(\frac{|\phi-1|}{\epsilon}, 1\right), & \text { if } \phi>1 .\end{cases} \\
S_{l i m}=\max \left(S_{Y_{k}}, S_{Z}, S_{Z^{\prime \prime}}\right)
\end{gathered}
$$


with $\phi$ being $Y_{k}, Z$ or $Z^{\prime \prime}$ and $\epsilon=0.1$. The final limiter $S_{\text {lim }}$ is taken as the maximum of the three limiters (Eq. 18)

Conservative artificial viscosity. At the end of the temporal iteration (i.e. after the two substeps of the TTGC scheme) and before the application of the boundary conditions, second-order derivatives are used to add artificial diffusion and filter the conservative variables when the sensor is activated to smooth the largest gradient. At node $i$, artificially diffused conservative variables are obtained from:

$$
\begin{aligned}
\left(\bar{\rho} \widetilde{Y_{k}}\right)_{i}^{A V} & =\left(\bar{\rho} \widetilde{Y_{k}}\right)_{i}+\frac{1}{V_{i}} \sum_{j \mid i \in \Omega_{j}}-\frac{V_{\Omega_{j}}}{n_{v}}\left(\left(\bar{\rho} \widetilde{Y_{k}}\right)_{\Omega_{j}}-\left(\bar{\rho} \widetilde{Y_{k}}\right)_{i}\right)\left(S_{\rho, \Omega_{j}}+S_{\text {lim, } \Omega_{j}}\right) \\
(\bar{\rho} \widetilde{u})_{i}^{A V} & =(\bar{\rho} \widetilde{u})_{i}+\frac{1}{V_{i}} \sum_{j \mid i \in \Omega_{j}}-\frac{V_{\Omega_{j}} \widetilde{u}_{\Omega_{j}}\left(\bar{\rho}_{\Omega_{j}}-\bar{\rho}_{i}\right) S_{\rho, \Omega_{j}}}{n_{v}} \\
(\bar{\rho} \widetilde{E})_{i}^{A V} & =(\bar{\rho} \widetilde{E})_{i}+\frac{1}{V_{i}} \sum_{j \mid i \in \Omega_{j}}-\frac{V_{\Omega_{j}}}{n_{v}}\left[\left(\bar{\rho} \widetilde{e_{s}}\right)_{\Omega_{j}}-\left(\bar{\rho} \widetilde{e_{s}}\right)_{i}+\widetilde{e_{c, \Omega_{j}}}\left(\bar{\rho}_{\Omega_{j}}-\bar{\rho}_{i}\right)\right]\left(S_{\rho, \Omega_{j}}+S_{l i m, \Omega_{j}}\right) \\
(\bar{\rho} \widetilde{Z})_{i}^{A V} & =(\bar{\rho} \widetilde{Z})_{i}+\frac{1}{V_{i}} \sum_{j \mid i \in \Omega_{j}}-\frac{V_{\Omega_{j}}}{n_{v}}\left((\bar{\rho} \widetilde{Z})_{\Omega_{j}}-(\bar{\rho} \widetilde{Z})_{i}\right)\left(S_{\rho, \Omega_{j}}+S_{l i m, \Omega_{j}}\right) \\
(\bar{\rho} \widetilde{Z})_{i}^{A V} & =(\bar{\rho} \widetilde{Z})_{i}+\frac{1}{V_{i}} \sum_{j \mid i \in \Omega_{j}}-\frac{V_{\Omega_{j}}}{n_{v}^{\Omega_{j}}}\left(\left(\bar{\rho} \widetilde{Z^{\prime 2}}\right)_{\Omega_{j}}-\left(\bar{\rho} \widetilde{Z}^{\prime 2}\right)_{i}\right)\left(S_{\rho, \Omega_{j}}+S_{l i m, \Omega_{j}}\right)
\end{aligned}
$$

where $S_{\rho, \Omega_{j}}=1 / n_{v}^{\Omega_{j}} \sum_{k \in \Omega_{j}} S_{\rho, k}$ and $S_{l i m, \Omega_{j}}=1 / n_{v}^{\Omega_{j}} \sum_{i \in \Omega_{j}} S_{l i m, i}$. Cell velocity equilibrium is assumed in Eqs. 20 and 21 during the diffusion of the conservative variables.

Non-conservative artificial viscosity. To limit the generation of acoustic waves during artificial diffusion (see Sec. 2.5.2), a non-conservative artificial viscosity [45] is used in this work. Energy $\rho E$ is not directly filtered using Eq. 21 when artificial viscosity is applied to the flow. Instead, $(\bar{\rho} \widetilde{E})^{A V}$ is computed from the artificially filtered pressure $p^{A V}$ and species density $\left(\bar{\rho} \widetilde{Y}_{k}\right)^{A V}$ and is introduced in the code using a splitting procedure, after the application of conservative artificial viscosity, and a relaxation formulation:

$$
\begin{aligned}
p_{i}^{A V} & =p_{i}+\frac{1}{V_{i}} \sum_{j \mid i \in \Omega_{j}}-\frac{V_{\Omega_{j}}}{n_{v}^{\Omega_{j}}}\left(p_{\Omega_{j}}-p_{i}\right)\left(S_{\rho, \Omega_{j}}+S_{l i m, \Omega_{j}}\right) \\
\frac{\partial \bar{\rho} \widetilde{e_{s}}}{\partial t} & =\kappa_{e}\left(\widetilde{e_{s}}-{\widetilde{e_{s}}}^{*}\right) H\left(0.9-Z^{t h}\right)
\end{aligned}
$$

where ${\widetilde{e_{s}}}^{*}={\widetilde{e_{s}}}^{*}\left(\left(\widetilde{\rho} \widetilde{Y_{k}}\right)^{A V}, p^{A V}\right)$ and $H$ is the Heaviside function. Stiff relaxation is computed $\left(\kappa_{e} \rightarrow \infty\right)$ to ensure $\widetilde{e_{s}}={\widetilde{e_{s}}}^{*}$ at the end of the iteration. Using a non-conservative method may lead to temperature error [40] and this procedure is then restricted to the strongly non-linear thermodynamic region $\left(Z_{t h}=p /(\rho r T)<0.9\right)$. For $Z_{t h}>0.9$, the conservative procedure is conserved. It was observed that temperature errors may be large if the non-conservative formulation is applied everywhere in the flow, especially if sub-grid scale terms are neglected. Compared with other numerical approaches $[26,31,33]$, this method is not oscillation free, but leads to a subsequent diminution of non-physical pressure noise, as shown below. It is expected to be applicable to other numerical scheme. The additional CPU cost compared with the fully conservative procedure is $5 \%$ on a $3 \mathrm{D}$ simulation.

\subsection{2 $1 D$ test-cases}

Simple qualitative test-cases are shown below to assess the performance of the numerical strategy used in this work. A 1D oxygen interface is advected in an open domain (non-reflecting boundary conditions on each side of the domain). The initial density profile $\rho(x)$ is given by:

$$
\rho(x)=\rho_{\max }+0.5\left(\rho_{\min }-\rho_{\max }\right)\left(1+\operatorname{erf}\left(\sqrt{6} \frac{x}{n_{x} \Delta x}\right)\right)
$$


where $\Delta x=0.01 \mathrm{~m}$ is the characteristic grid spacing, $\rho_{\max }=1000 \mathrm{~kg} \mathrm{~m}^{-3}$ and $\rho_{\min }=100 \mathrm{~kg} \mathrm{~m}^{-3}$. The initial velocity is constant and set to $10 \mathrm{~m} \mathrm{~s}^{-1}$. Three values of the parameter $n_{x}$ are studied: 2, 4 and 6, that approximatively correspond to 2, 4 and 6 cells in the density gradient.

Conservative artificial viscosity. The interface is first advected without artificial viscosity (continuous lines on Fig. 4). As expected from [76], oscillations are visible on density that leads to strong overshoot of temperature, especially for $n_{x}=2$. The under-resolution of the interface produces acoustic disturbances, visible on pressure and velocity, that propagate in the domain. These fluctuations are strongly reduced as $n_{x}$ is increased and are no longer noticeable for $n_{x}=6$. Both density and temperature overshoot are reduced when artificial viscosity is added (Circles in Fig. 4). Using a fully conservative artificial viscosity leads to the generation of acoustic waves during artificial diffusion $[31,45]$. These waves may artificially promote the generation of an acoustic mode [40], and should therefore be limited in the case of combustion instabilities simulation.

a)
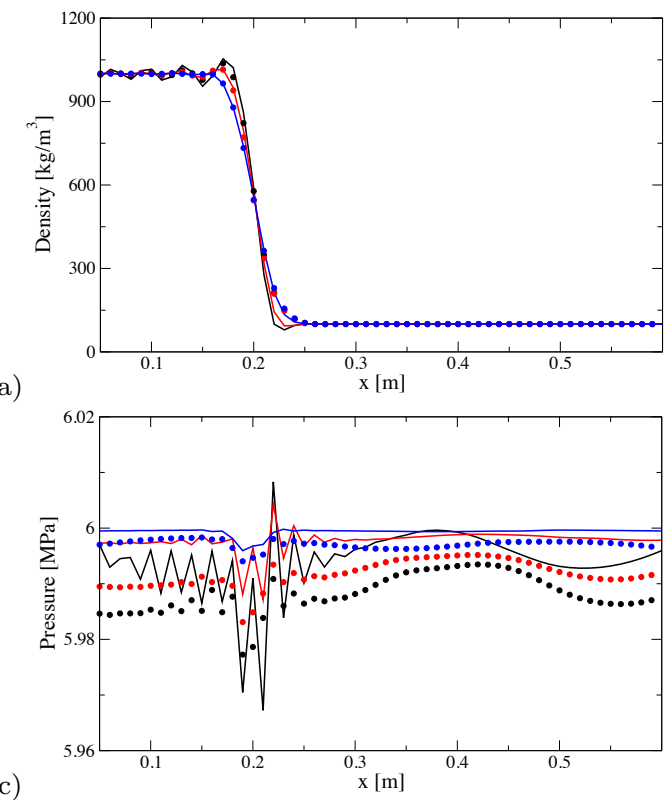

b)
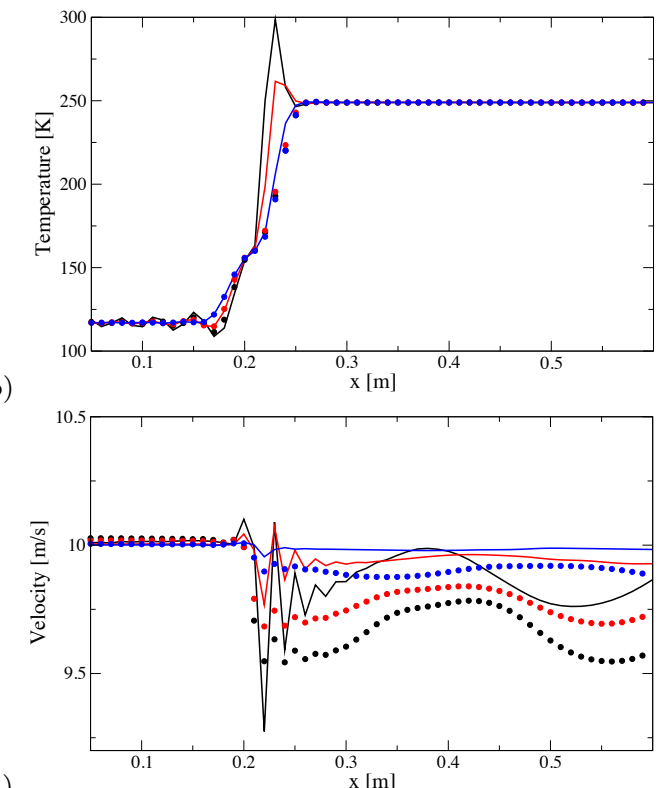

Fig. 4 Advection of an oxygen density interface after $20 \mathrm{~ms}$. (a) Density, (b) temperature, (c) pressure and (d) velocity. Black: $n_{x}=2$, red: $n_{x}=4$, blue: $n_{x}=6$. Continuous lines: no artificial viscosity, circles: conservative artificial viscosity.

Non-conservative artificial viscosity. Figure 5 compares instantaneous density, temperature, pressure and velocity field after $20 \mathrm{~ms}$ with conservative (circles on Fig. 5) and non-conservative artificial viscosity (continuous lines on Fig. 5). While density and temperature are virtually not impacted by the formulation, pressure fluctuations are strongly reduced with the non-conservative procedure. Limited disturbances are still noticeable in the interface, but do not propagate downstream contrary to the fully conservative formulation. The acoustic noise produced by the stabilisation method is strongly reduced when the non-conservative formulation is used.

Multi-species test case. A test case with 2 species, $\mathrm{H}_{2}$ and $\mathrm{O}_{2}$, initally at $100 \mathrm{~K}$ and 60 bar is now considered. The initial oxygen mass fraction profile $Y_{O_{2}}(x)$ is given by:

$$
Y_{O_{2}}(x)=0.5\left(1-\operatorname{erf}\left(\sqrt{6} \frac{x}{n_{x} \Delta x}\right)\right)
$$

where $\Delta x=0.01 \mathrm{~m}$ is the characteristic grid spacing. The initial velocity is constant and set to 10 $\mathrm{m} \mathrm{s}^{-1}$. Three values of the parameter $n_{x}$ are studied: $n_{x}=10^{-5}, 2$ and 4 , that approximatively correspond to 0,2 and 4 cells in the density gradient. The resulting density field varies from 
a)

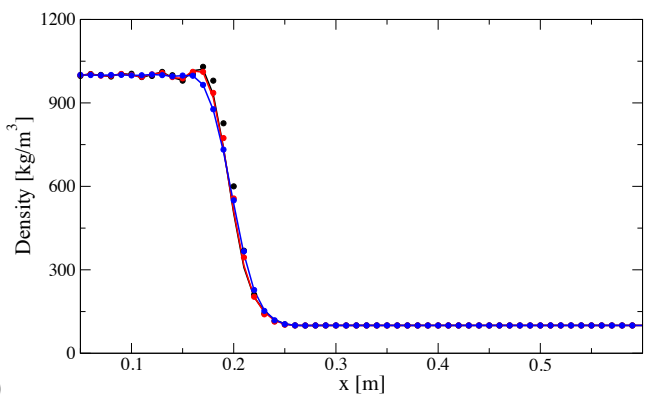

c)

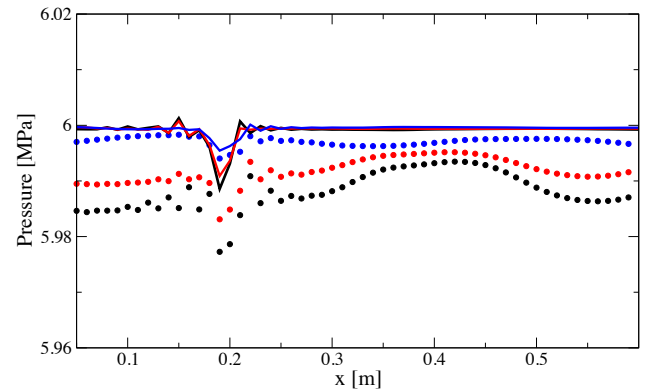

b)
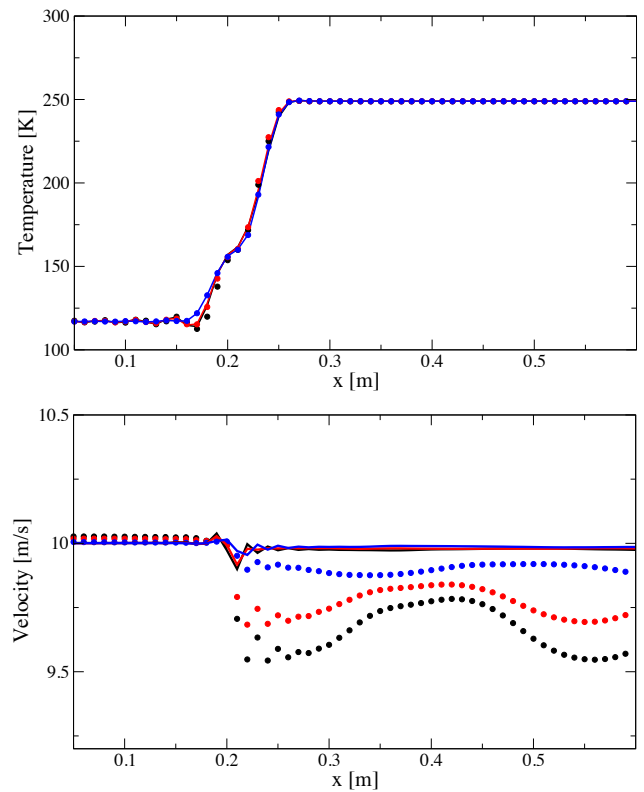

Fig. 5 Advection of an oxygen density interface after $20 \mathrm{~ms}$. (a) Density, (b) temperature, (c) pressure and (d) velocity. Black: $n_{x}=2$, Red: $n_{x}=4$, Blue: $n_{x}=6$. Circles: conservative artificial viscosity, continuous lines: nonconservative artificial viscosity.

$\rho_{\max }=1104 \mathrm{~kg} \mathrm{~m}^{-3}$ and $\rho_{\min }=14.5 \mathrm{~kg} \mathrm{~m}^{-3}$.

Figure 6 compares instantaneous density, temperature, pressure and velocity field after $20 \mathrm{~ms}$ with conservative (circles on Fig. 6) and non-conservative artificial viscosity (continuous lines on Fig. 6). Similarly to what is shown on Fig. 5, density is virtually not impacted using either the conservative or non conservative formulation. Conservation errors on energy now lead to a departure of $20 \mathrm{~K}$ to $40 \mathrm{~K}$ with the fully conservative formulation. These conservation errors are however restricted to the cold non-linear region $\left(Z^{\text {th }}<0.9\right.$, Eq. 25). Pressure fluctuations are reduced with the non-conservative formulation even though limited disturbances are still noticeable in the interface. The acoustic noise produced by the stabilisation method is strongly reduced when the non-conservative formulation is used. This strategy is retained for the $3 \mathrm{D}$ simulations.

Influence of $\xi$. The impact of the threshold coefficient $\xi$ is shown in Fig. 7 for the multi-species case shown previously (Eq. 27) with $n_{x}=2$. Imposing a value of $\xi$ larger than 0 cancels the detection of under-resolved oscillations with the smallest amplitudes. Only the largest relative density oscillations are then detected by the sensor. As shown in Fig. 7, increasing $\xi$ limit numerical dissipation and sharpen the density profile, especially for low density values, at the cost of increased numerical noise. In particular, for $\xi \geq 3$, important acoustic perturbations are noticed. An estimation of the thickness is $\delta_{\text {interface }}=\left(\left(\rho_{\max }-\rho_{\min }\right) / \Delta x\right)(\nabla \rho)_{\max }$. It gives $4.21,3.48,3.19$ and 3.13 for $\xi=0,1$, 3 and 10 , respectively. 
a)

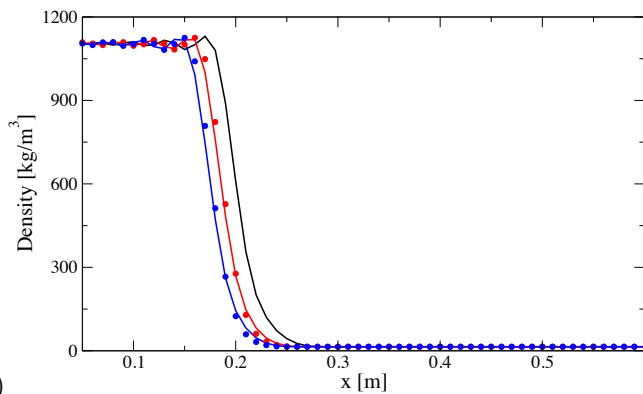

c)

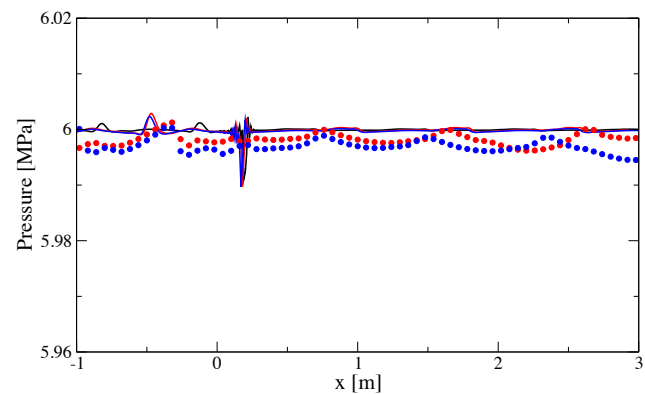

b)

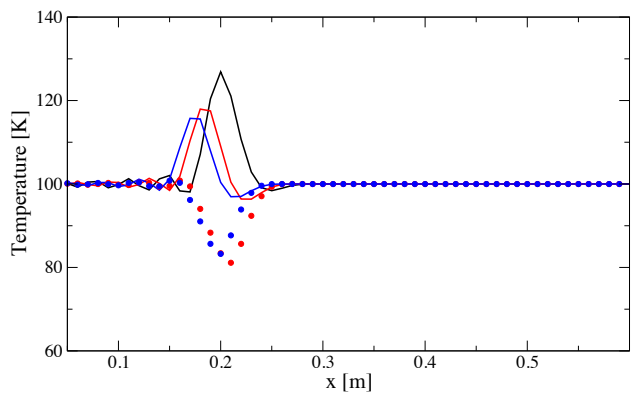

d)

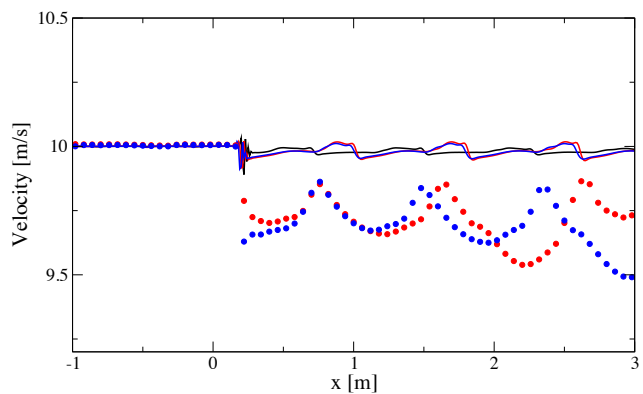

Fig. 6 Advection of a $\mathrm{H}_{2} / \mathrm{O}_{2}$ interface after $20 \mathrm{~ms}$. (a) Density, (b) temperature, (c) pressure and (d) velocity. Black: $n_{x}=\epsilon$, Red: $n_{x}=2$, Blue: $n_{x}=4$. Circles: conservative artificial viscosity, continuous lines: non-conservative artificial viscosity. The conservative calculation is not stable for $n_{x}=\epsilon$.

a)
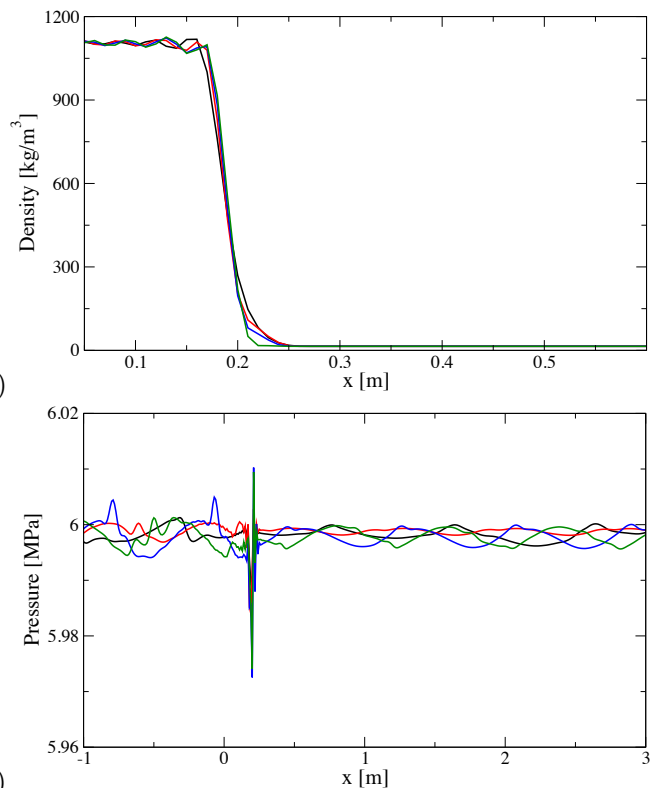

b)

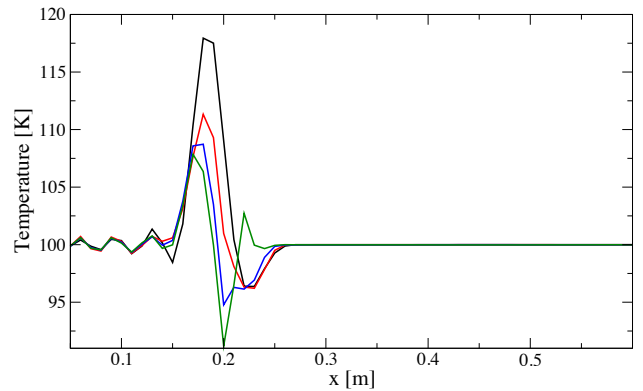

d)

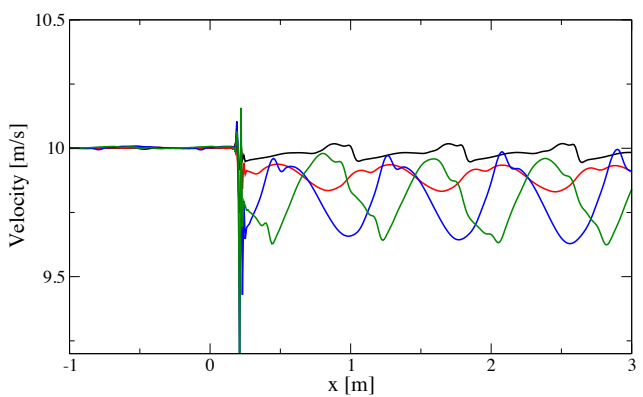

Fig. 7 Advection of a $\mathrm{H}_{2} / \mathrm{O}_{2}$ interface after $20 \mathrm{~ms}$. Influence of threshold coefficient $\xi$ for $n_{x}=2$. (a) Density, (b) temperature, (c) pressure and (d) velocity. Black: $\xi=0.0$, Red: $\xi=1.0$, Blue: $\xi=3.0$, Green: $\xi=10.0$.

\section{Numerical and experimental configurations}

\subsection{Experimental setup}

The Mascotte experimental configuration of ONERA [80], which has been extensively used for experimental studies of cryogenic combustion $[4,8]$, is considered here. The present simulations reproduce cases A60, C60 and G2, corresponding to transcritical flames [81,82]. A single coaxial injector produces a dense oxygen stream (i.e. liquid-like oxygen) at low velocity (less than $10 \mathrm{~m} / \mathrm{s}$ ), surrounded by a high-velocity gaseous hydrogen (cases A60 and C60) or methane (case G2) stream 
(more than $100 \mathrm{~m} / \mathrm{s}$ ), in a chamber at supercritical-pressure for both reactants (60 bar for case A60 and C60, 56 bar for case G2). Cases A60 and C60 differ only by the hydrogen mass flow rate, which is 50\% larger in the A60 case. Oxygen under transcritical conditions is injected at $83 \mathrm{~K}$, well below its critical value, while hydrogen and methane, injected at $300 \mathrm{~K}$ are supercritical and gaseous, respectively (see Tab. 1). Under such conditions, the density of oxygen $\left(\rho_{\mathrm{O}_{2}}=1200 \mathrm{~kg} \cdot \mathrm{m}^{-3}\right)$ is much larger than that of hydrogen $\left(\rho_{H_{2}}=5.25 \mathrm{~kg} \cdot \mathrm{m}^{-3}\right)$ and methane $\left(\rho_{C H_{4}}=39.5 \mathrm{~kg} \cdot \mathrm{m}^{-3}\right)$. The momentum flux ratio $\mathrm{J}=\left(\rho_{f} u_{f}^{2}\right) /\left(\rho_{\mathrm{O}_{2}} u_{\mathrm{O}_{2}}^{2}\right)$, a critical parameter that drives coaxial flames length and spreading rate [8], is close to 7 and 14 for cases $\mathrm{C} 60$ and A60, respectively, and its value is higher in case G2 because the density of methane is higher than that of hydrogen [8]. It should be noticed that in all cases mixture is rich so that hydrogen or methane is always in excess.

\begin{tabular}{|c|c|c|c|c|c|c|c|}
\hline Species & $\mathrm{H}_{2}$ & $\mathrm{CH}_{4}$ & $\mathrm{O}_{2}$ & $\mathrm{H}_{2} \mathrm{O}$ & $\mathrm{OH}$ & $\mathrm{CO}$ & $\mathrm{CO} 2$ \\
\hline$T_{C}[\mathrm{~K}]$ & 33.0 & 191 & 155 & 647 & 105 & 133 & 304 \\
\hline$p_{C}[\mathrm{MPa}]$ & 1.28 & 4.60 & 5.04 & 22.0 & 7.09 & 3.50 & 7.38 \\
\hline
\end{tabular}

Table 1 Species critical temperature $\left(T_{C}\right)$ and pressure $\left(p_{C}\right)$.

\subsection{Computational domain, mesh and boundary conditions}

The computational domain is sketched in Fig. 8a and reproduces accurately the geometry of the experimental chamber. The section of the combustion chamber is square-shaped (50 $\mathrm{mm} \times 50 \mathrm{~mm})$, and the domain length is $400 \mathrm{~mm}$. The three-dimensional simulations are performed on a mesh containing 2,000,000 points and 11,500,000 tetrahedra. The smallest cell is located at the tip of the separator between oxygen and fuel, meshed with 5 cells in its thickness (Fig. 8b). The mesh is progressively coarsened downstream, where the flow and flame structures are less sharp.

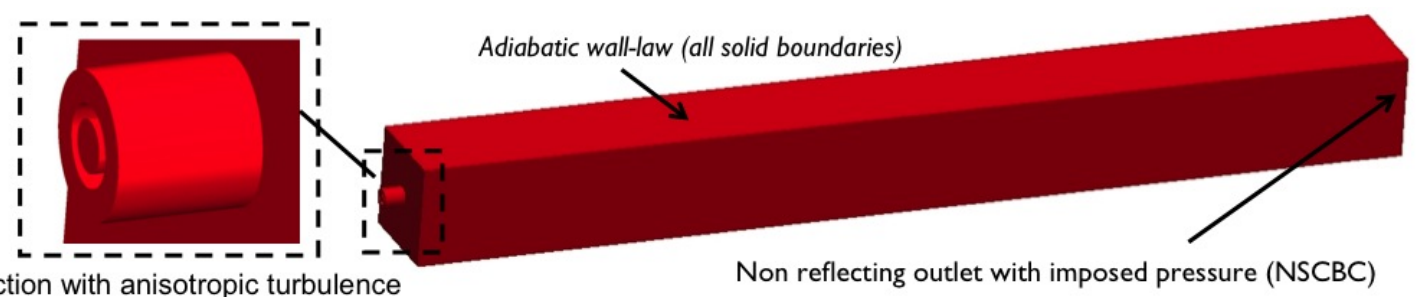

(t) $3 \%$ at the

a) Center of the pipes)

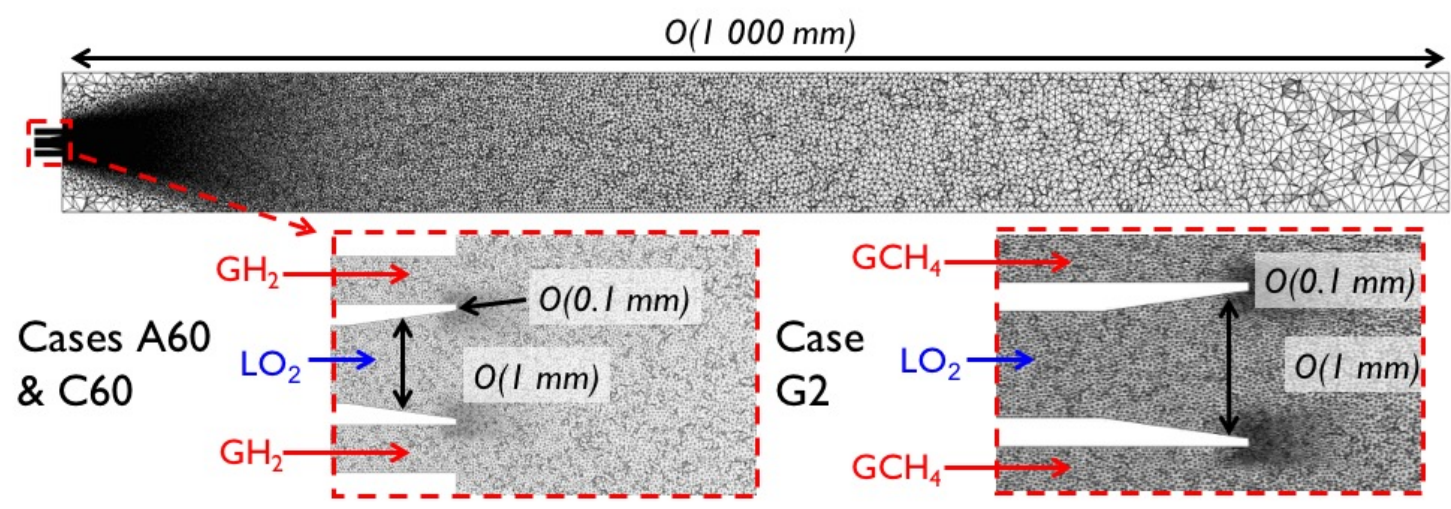

b)

Fig. 8 Computational domain. (a) Sketch of the computational domain, providing details about the boundary conditions. (b) Longitudinal slice of the mesh and zoom in the near injector region.

Walls are treated using adiabatic slip wall-law boundary condition, which includes wall functions $[83,84]$. The inlet and outlet conditions are both set with non-reflecting characteristic boundary 
conditions, with a relaxation on the pressure at the exit boundary condition [85]. Turbulent velocity fluctuations are superimposed to the bulk flow at the injection on both oxygen and fuel streams [86, 87] following the injection profiles plotted in Fig. 9 and a Passot-Pouquet spectrum. The same grid distribution, boundary conditions and numerics are used for all the simulations. Only mass flow rates and injected species are changed to correspond to the case considered.

a)

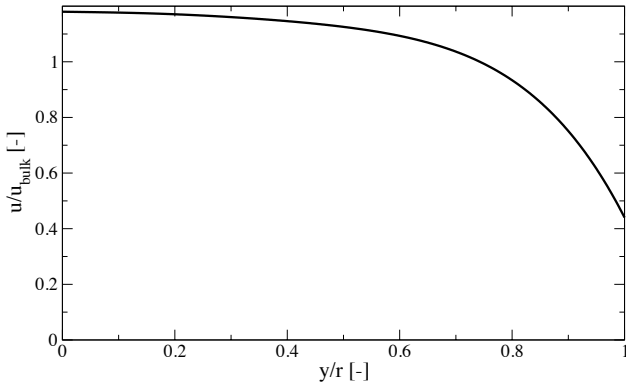

b)

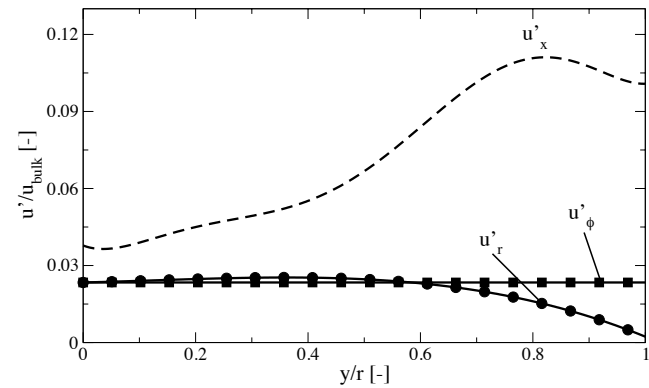

Fig. 9 Injection velocity profiles. (a) Mean axial velocity $u$, (b) Axial $\left(u_{x}^{\prime}\right)$, radial $\left(u_{r}^{\prime}\right)$ and azimuthal $\left(u_{\phi}^{\prime}\right)$ rms velocities. $u_{\text {bulk }}$ is the bulk velocity at inlet.

\subsection{Averaging procedure and computational time}

All the statistics presented in this paper are obtained using a Reynolds averaging procedure. For all simulations, the calculations are run for 1 convective time (based on the oxygen injection velocity and the flame length) before statistics are gathered. In order to asses the required computational time for a proper comparison between the cases, case A60 is computed on a coarse grid (see Sec. 4.1 for details) for 2 averaging times $\tau_{a v}$. Mean and rms profiles for $\tau_{a v}=100 \mathrm{~ms}$ (black lines) and $\tau_{a v}=50 \mathrm{~ms}$ (red lines) are shown on Fig. 10. It shows that an averaging time of $50 \mathrm{~ms}$ is sufficient, except at $\mathrm{x}=20 \mathrm{~d}$ and $\mathrm{r}<10 \mathrm{~mm}$ where little departures are noticeable. This is due to the very low velocity in this region, close to zero. In the following, averages are then performed over $50 \mathrm{~ms}$.

Averaging and computational times are gathered in Tab. 2.

\begin{tabular}{|c|c|c|c|}
\hline Case & $\tau_{a v}[\mathrm{~ms}]$ & Number of temporal iterations & CPU time $[\mathrm{h}]$ \\
\hline C60 & 50 & $4.810^{6}$ & 180000 \\
\hline A60 coarse mesh & 50 & $3.110^{6}$ & 35000 \\
\hline A60 & 50 & $4.810^{6}$ & 180000 \\
\hline G2 & 50 & $2.910^{6}$ & 155000 \\
\hline
\end{tabular}

Table 2 Averaging times and CPU cost of the simulations. Simulations are run on Intel Broadwell processors at $2.6 \mathrm{GHz}$ and with 2240 cores for the fine grids and 560 cores for the coarse grid.

\section{Validations}

\subsection{Numerical strategy for LES}

The numerical approaches are tested within the LES framework (i.e. with sub-grid scale models and combustion models activated) and on a realistic case, the case A60. In order to highlight the possible influence of numerics on the results, a coarse grid is used in this section. The impact of numerics observed here is expected to be strongly reduced as the grid is refined. The characteristic cell size is increased by a factor 1.5 over the whole domain, keeping the same distribution than the original grid (referred as the fine grid herein). The coarse grid contains 750,000 points and 
a)

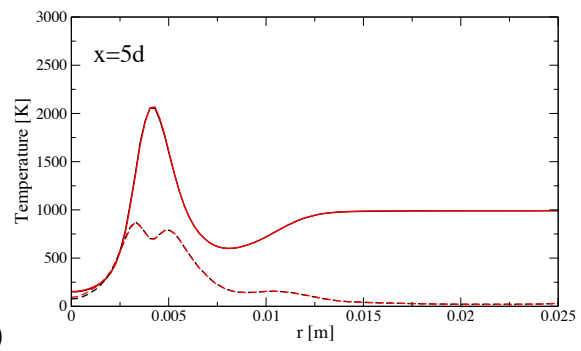

b)

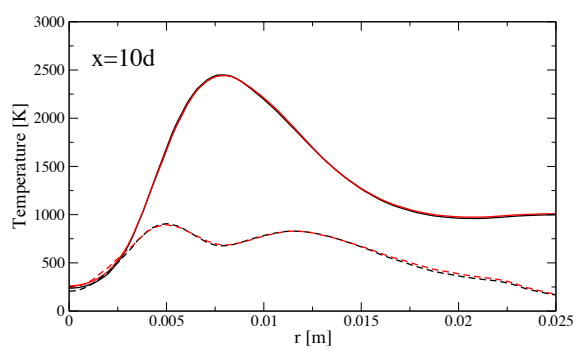

e)
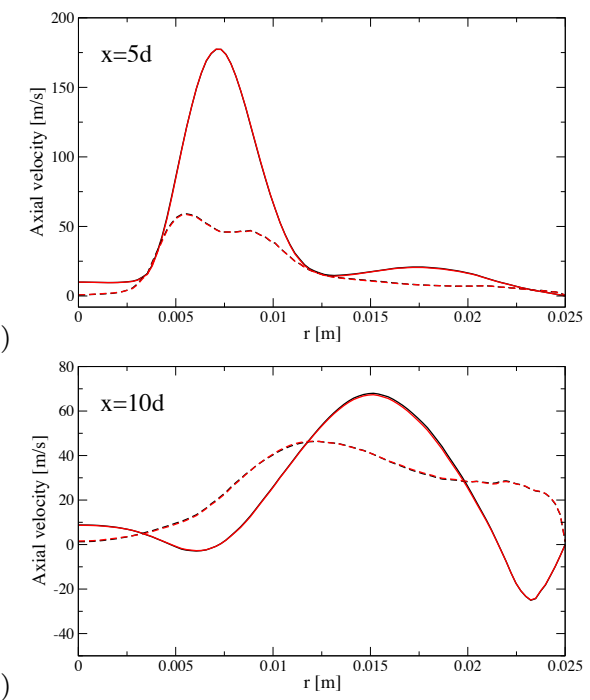

f)

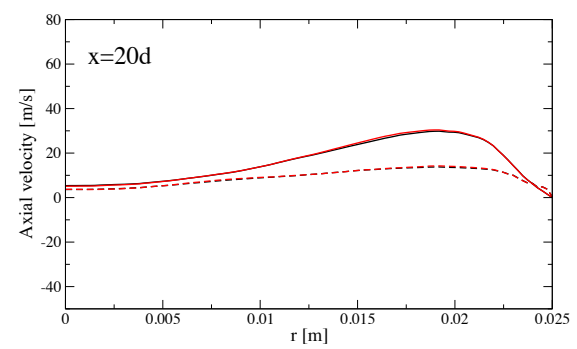

c)

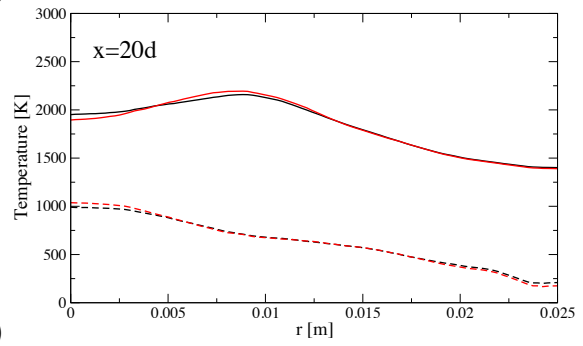

g)

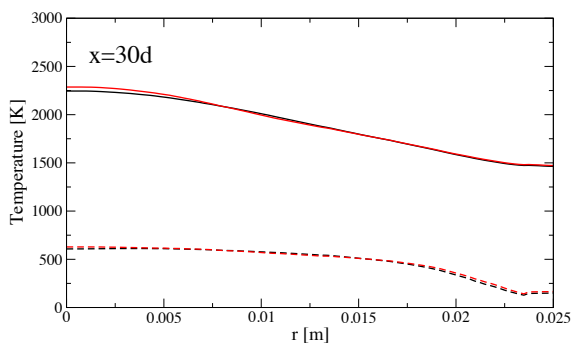

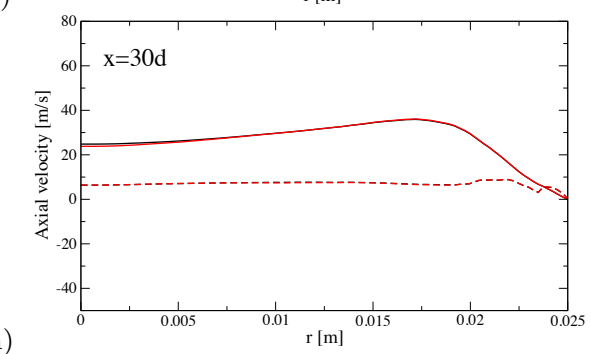

Fig. 10 Influence of averaging time on statistics for case A60. Radial profiles of temperature (plots a, b, c and d) and axial velocity (plots e, f, g and h) at $x=5 d, x=10 d, x=20 d$ and $x=30 d$ from the injector. Black lines: averaging time of $100 \mathrm{~ms}$, red lines: averaging time of $50 \mathrm{~ms}$. Continuous lines correspond to mean values, dashed lines are rms data.

4,400,000 tetrahedra and the injector tip resolution is consequently reduced to 3 cells . Radial profiles of mean and rms temperature and axial velocity at 3 different axial positions along the flame are plotted in Fig. 11. Conservative and non conservative approaches are plotted in black and red, respectively. The parameter $\xi$ is set to 0.0 for these two simulations. Only small differences can be seen between the two approaches, the flame length of the non conservative one being slightly shorter. As expected from the 1d tests cases, the conservative procedure produces more pressure fluctuations than the non conservative one (Fig. 12), especially near the injector lips, were density gradients and artificial viscosity are the largest. For such a simulation, these fluctuations remains limited and have only a small influence on the jet destabilization, the initial spreading rate being slightly increased in the non-conservative procedure compared with the conservative one. The non-conservative procedure is however chosen to limit possible artificial pressure disturbance in combustion instabilities simulations. Eventually, the impact of numerics is investigated by increasing the parameter $\xi$ from $\xi=0$ to $\xi=1, \xi=3$ and $\xi=10$. It is found that the numerical parameter $\xi$ can be increased up to $\xi=3$ with no major changes in the global results to further reduce the amount of artificial dissipation in practical simulations as shown in Fig. 11. In particular, maximum temperature is decreased at $x=5 \mathrm{~d}$ and $x=10 \mathrm{~d}$ as $\xi$ is increased, especially for the case $\xi=10$. On the contrary, axial velocity at $x=5 \mathrm{~d}$ is virtually unaffected by the numerical change, which seems 
to indicate that numerics impact combustion in this case. To investigate this aspect, radial profiles of $Z$ and $Z^{\prime \prime}$ are plotted in Fig. 13. At $x=5 \mathrm{~d}$, all the mixture fraction profiles are close to each other. The variance of $Z$ is however slightly larger for the cases with $\xi=3$ and $\xi=10$ compared with the other, which could explain the lower temperature predicted in these cases in the near injector region. Numerical dissipation also increases the flame spreading rate. This is observed at $x=10 \mathrm{~d}$ on mixture fraction, temperature and velocity profiles.

a)

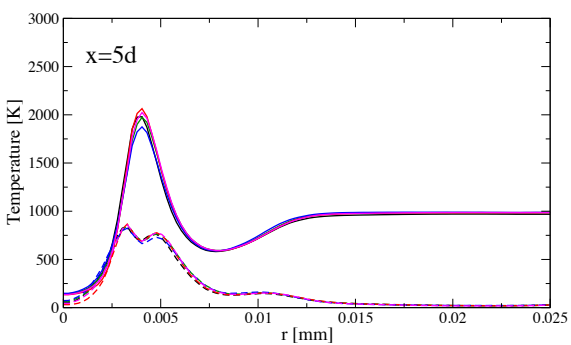

b)

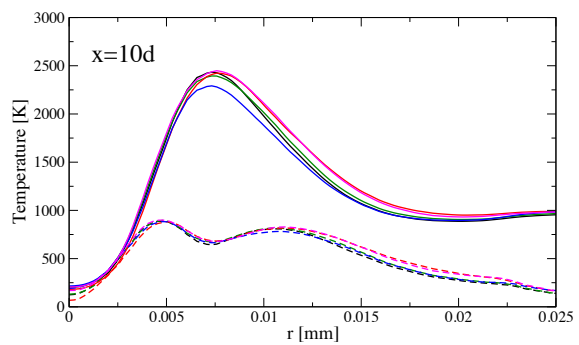

c)
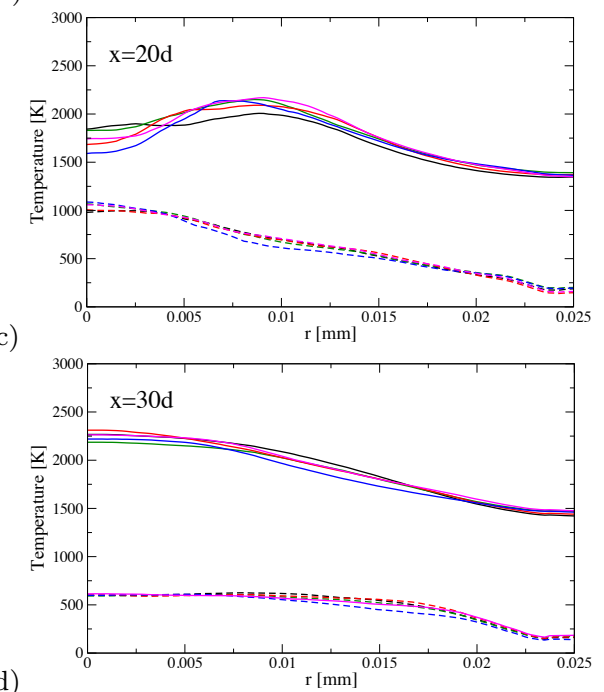

e)
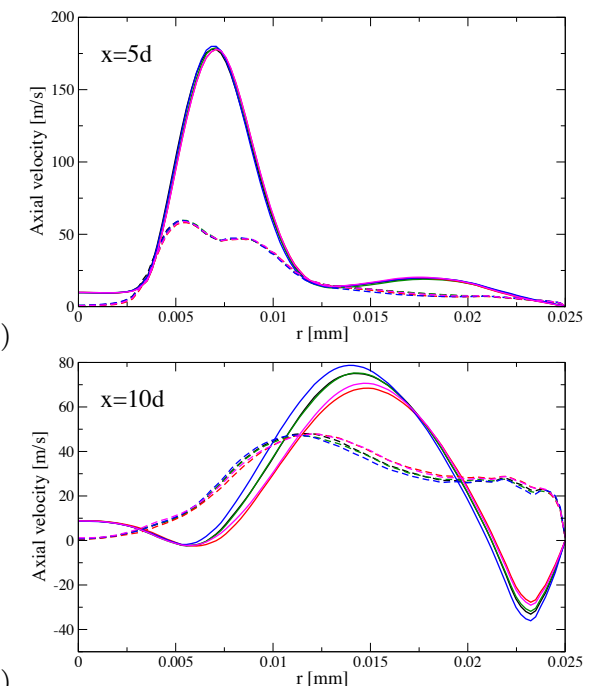

)

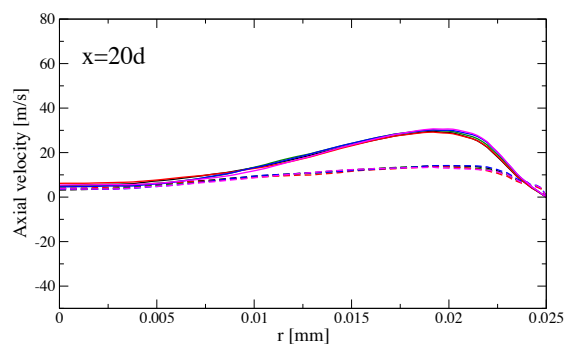

g)

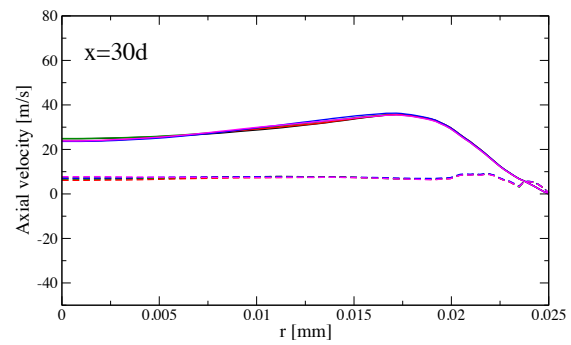

Fig. 11 Influence of numerical parameters on case A60. Radial profiles of temperature (plots a, b, c and d) and axial velocity (plots e, $\mathrm{f}, \mathrm{g}$ and $\mathrm{h}$ ) at $x=5 d, x=10 d, x=20 d$ and $x=30 d$ from the injector. Black lines: fully conservative artificial viscosity with $\xi=0.0$ in Eq. 14 , red lines: non conservative artificial viscosity with $\xi=0.0$, magenta lines: non conservative artificial viscosity with $\xi=1.0$, green lines: non conservative artificial viscosity with $\xi=3.0$, blue lines: non conservative artificial viscosity with $\xi=10.0$. Continuous lines correspond to mean values, dashed lines are rms data.

\subsection{Mesh convergence}

The influence of the grid resolution in assessed performing case A60 on the coarse grid depicted in Sec. 4.1. Longitudinal slices of instantaneous temperature, axial velocity, density and oxygen mass fraction fields are shown in Fig. 14 for the coarse and the fine grids, respectively. Flows are similar, with the same flame topology and dynamics. 
a)
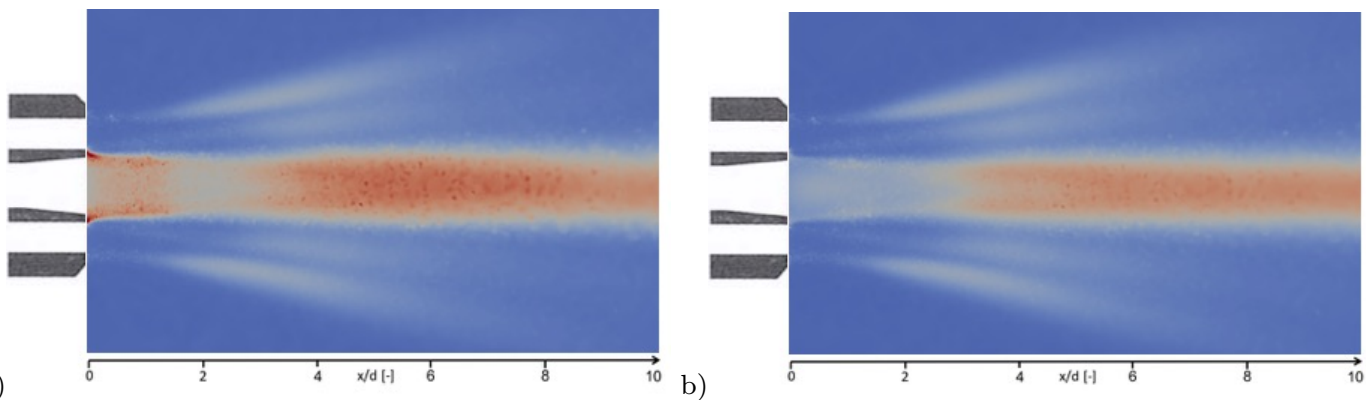

Fig. 12 Influence of numerical parameters on case A60. Longitudinal slices of pressure rms (blue: 0 ; red: 0.5 bar). (a) Conservative artificial viscosity, (b) Non conservative artificial viscosity.

a)
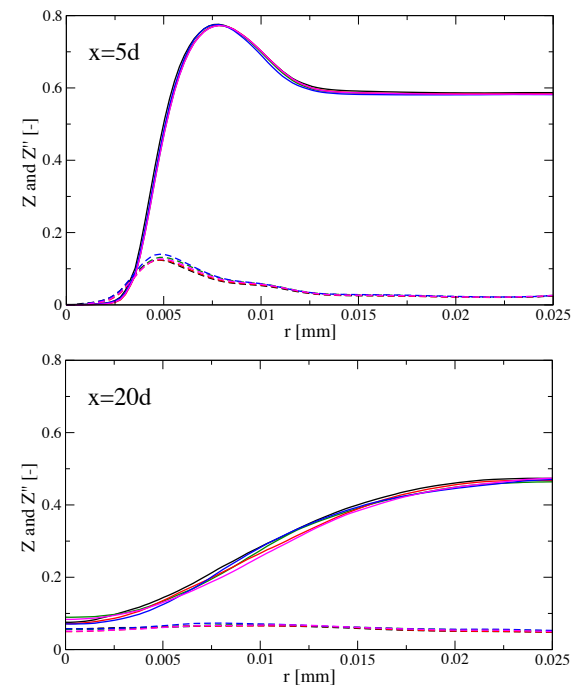

c)
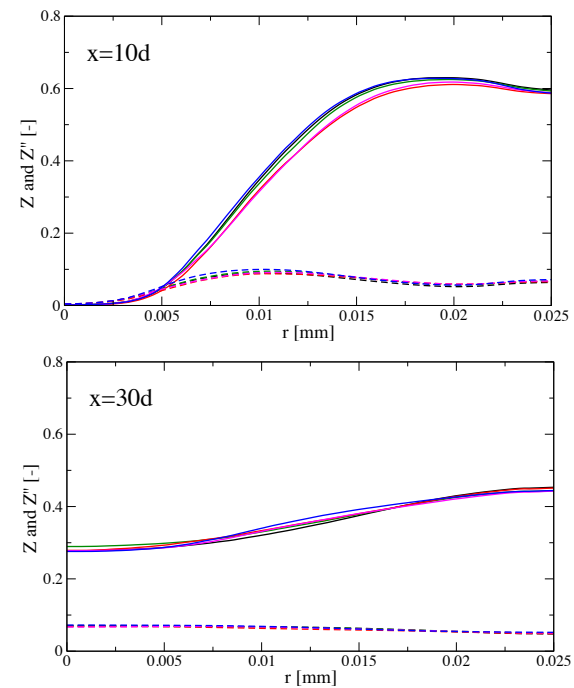

Fig. 13 Influence of numerical parameters on case A60. Radial profiles of mean mixture fraction $Z$ (continuous lines) and its variance $Z^{\prime \prime}$ (dashed lines) at $x=5 d, x=10 d, x=20 d$ and $x=30 d$ from the injector. Black lines: fully conservative artificial viscosity with $\xi=0.0$ in Eq. 14, red lines: non conservative artificial viscosity with $\xi=0.0$, magenta lines: non conservative artificial viscosity with $\xi=1.0$, green lines: non conservative artificial viscosity with $\xi=3.0$, blue lines: non conservative artificial viscosity with $\xi=10.0$.

Radial profiles of temperature and axial velocity at 3 different axial positions along the flame are plotted in Fig. 15. Agreement between the two meshes is satisfactory, both in terms of mean and rms values, validating the grid chosen for the study. In the same way, radial profiles of $Z$ and $Z$ " are similar for the two meshes as shown in Fig. 16. Variance is slightly higher for the coarser mesh, as expected. The strong departure on $Z$ profiles at $\mathrm{x}=20 \mathrm{~d}$ and $\mathrm{r}<0.01 \mathrm{~m}$ is due to limited statistics in this region where the velocity is close to zero (Fig. 16g). 

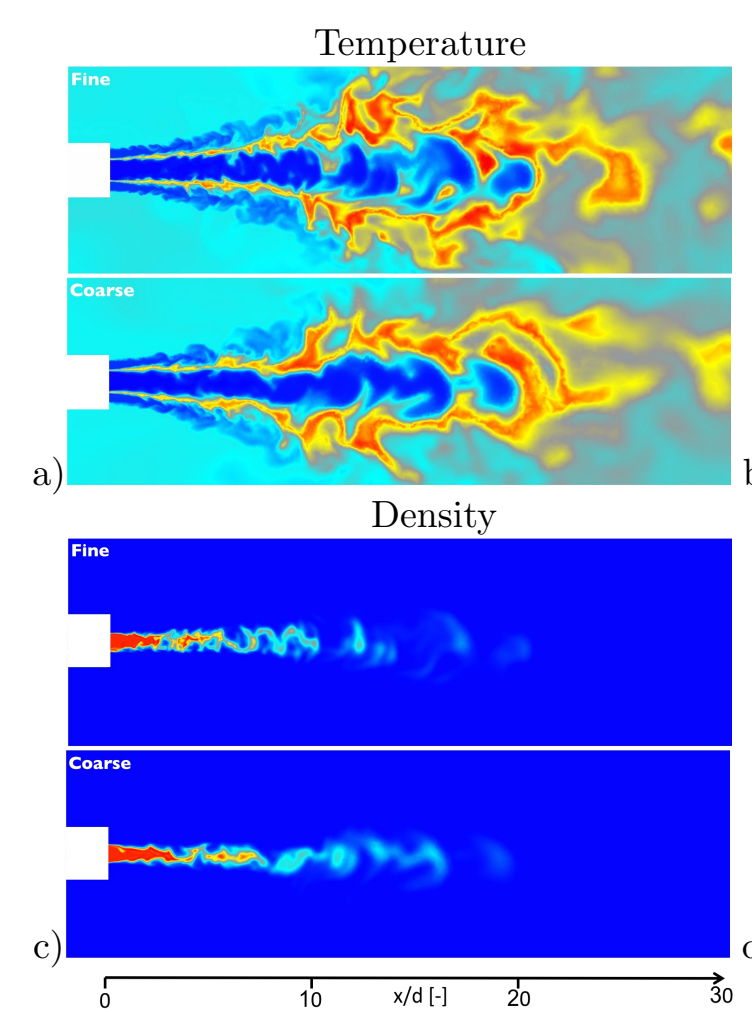

b)

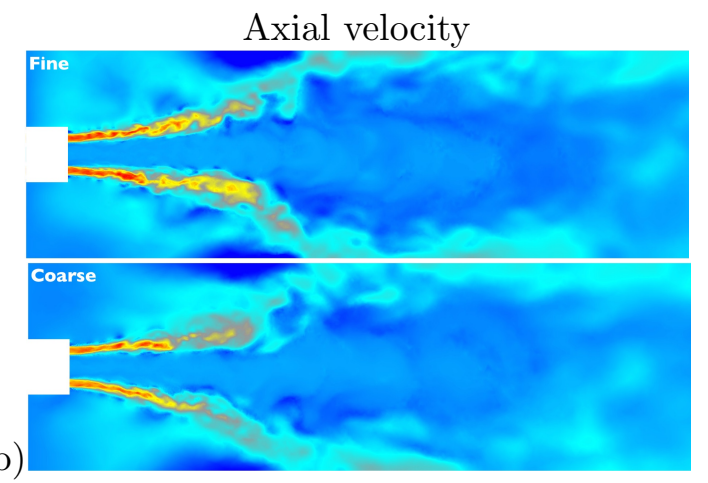

$\mathrm{O}_{2}$ mass fraction

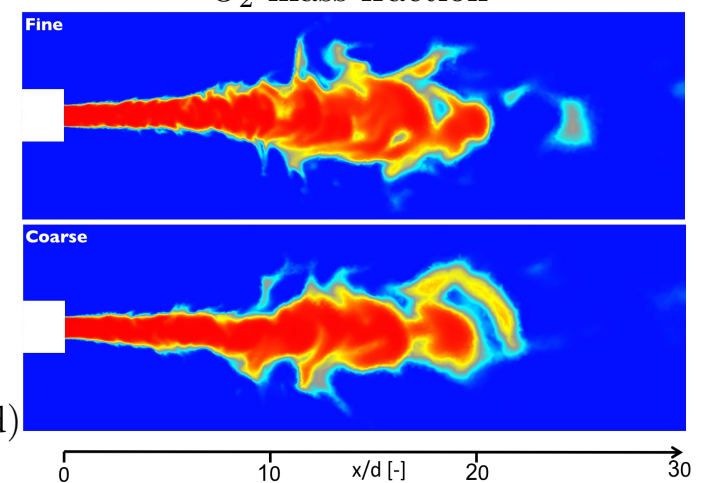

Fig. 14 Mesh convergence on case A60. Longitudinal slice of instantaneous (a) temperature, (b) axial velocity, (c) density and (d) oxygen mass fraction on fine and coarse grids. Lowest values are in blue, largest ones are in red. The color map is the same for both grids.

\subsection{Comparison with experimental data}

\section{Comparison between reconstructed $\mathrm{OH}^{*}$ emission signal, $\mathrm{OH}$ mass fraction and tem- perature fields}

For the very high pressure cryogenic cases simulated here, available experimental results essentially consist in $\mathrm{OH}^{*}$ emission signals $[88,82]$. In order to accurately compare experimental and numerical results, $\mathrm{OH}^{*}$ emission should be included in the simulation. This is attempted here using the partial equilibrium method proposed by Fiala and Sattelmayer [89]:

$$
\left[X_{O H^{*}}\right]=\left[X_{O H}\right] \exp \left(\frac{-\Delta g_{m f}^{0}}{R T}\right)=\rho \frac{Y_{O H}}{W_{O H}} \exp \left(\frac{-\Delta g_{m f}^{0}}{R T}\right)
$$

where $\left[X_{\mathrm{OH}^{*}}\right]$ is the molar concentration of $\mathrm{OH}^{*},\left[X_{\mathrm{OH}}\right]$ the molar concentration of $\mathrm{OH}, W_{O H}$ the molar mass of species $\mathrm{OH}$ and $\Delta g_{m f}^{0}$ is the difference in the molar Gibbs standard state enthalpy of formation. To account for flame / turbulence interaction, a $\beta$-pdf is used to filter $Y_{O H^{*}}$ mass fraction, similarly to what is done for species:

$$
\overline{\left[X_{O H^{*}}\right]}=\overline{\rho \frac{Y_{O H}}{W_{O H}} \exp \left(\frac{-\Delta g_{m f}^{0}}{R T}\right)}=\bar{\rho} \frac{\widetilde{Y_{O H^{*}}}\left(\widetilde{Z}, \widetilde{Z^{\prime \prime 2}}\right)}{W_{O H}}
$$

Instantaneous fields of $\mathrm{OH}^{*}$ concentration, $\mathrm{OH}$ mass fraction and temperature are shown in Fig. 17 for case $\mathrm{A} 60$. $\mathrm{OH}$ mass fraction and $\mathrm{OH}^{*}$ concentration fields are very close from each other: the two fields show signal at the same position, only limited amplitude departures are noticeable. Comparing temperature fields with $\mathrm{OH}$ field indicates that high temperature (greater than 2000 for case A60) may also be suited to get the flame position.

\section{Comparison with experimental visualizations}


a)

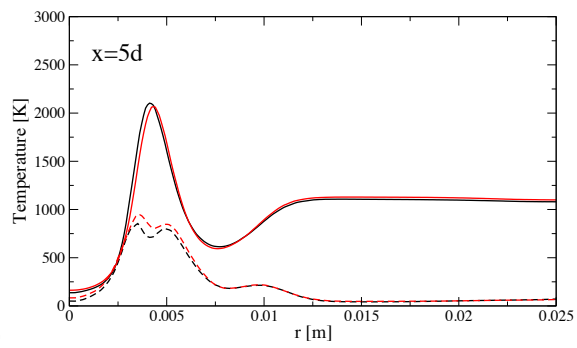

b)

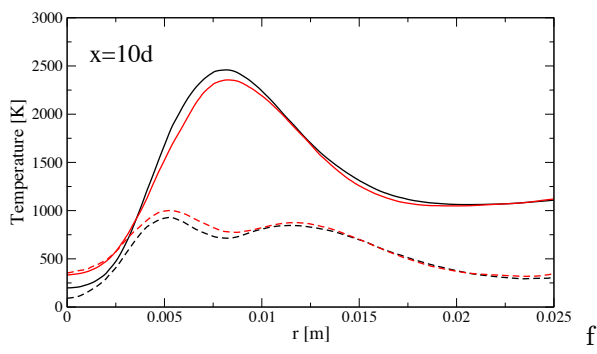

e)
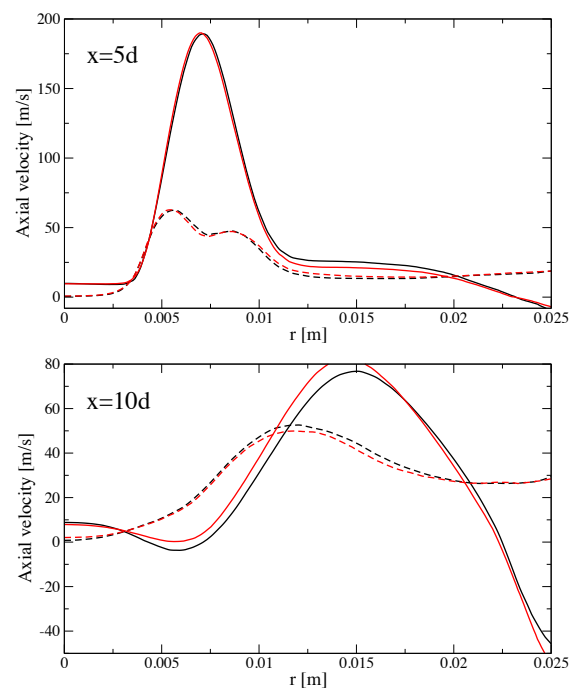

f)

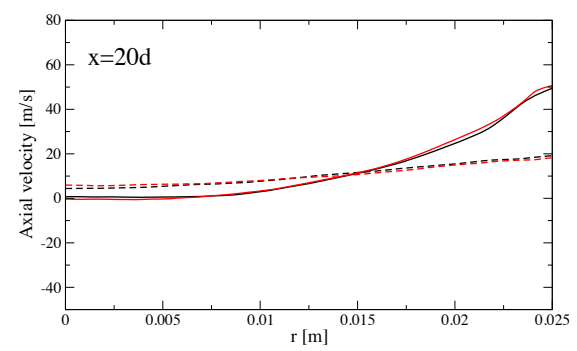

c)

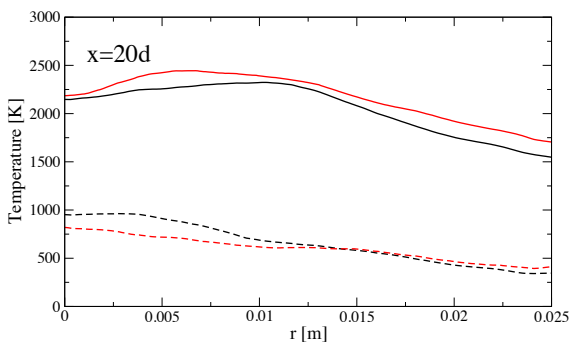

g)

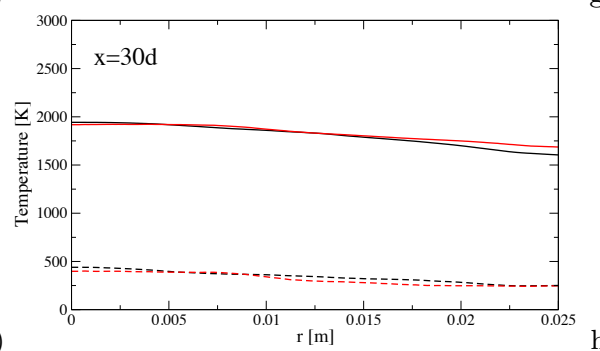

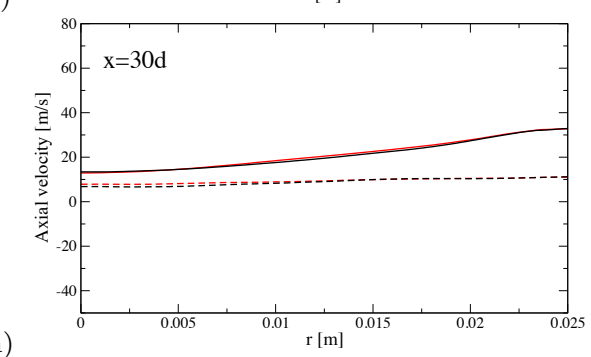

Fig. 15 Mesh convergence on case A60. Radial profiles of temperature (plots a, b and c) and axial velocity (plots $\mathrm{d}$, e and f) at $x=10 d, x=20 d$ and $x=30 d$ from the injector. Black line: coarse grid, red line: fine grid. Continuous lines correspond to mean values, dashed lines are rms data.

Comparisons of the numerical results with Abel transformed experimental $\mathrm{OH}^{*}$ emission (which estimate longitudinal cuts from integrated signals) from Juniper et al. [88] and Singla et al. [82] are proposed in Fig. 18 for cases C60, A60 and G2. For each simulated case, mean $\mathrm{OH}^{*}$ concentration field from LES is shown in the top and experimental visualization is shown in the bottom. For an easier comparison of flame position, the location of the maximum emission from experiment is plotted in dashed lines. Experimental results for case C60 show a strong asymmetry between the lower and upper part of the visualization, thus two lines are plotted here, showing the top (in red) and bottom (in black) experimental flame position. Very good agreements between experiments and numerics are obtained. In particular, the global flame positions are properly retrieved, with a good initial spreading angle and a correct position of the maximum spreading. For case C60, the position of maximum emission lies between the top and bottom experimental lines, the flame position seems then properly captured. In specific, in agreement with the experiments, the flame is not closed at the end of the window for case C60 while it is closing at the end of the visualization window for case A60 and it is terminated for case G2, with a good agreement in terms of flame length. However, $\mathrm{OH}^{*}$ emission amplitudes are in poor agreements between LES and experiments. In particular, maximum emission is found at $\mathrm{x}=12 \mathrm{~d}$ for cases $\mathrm{A} 60$ and $\mathrm{C} 60$, while it is located between $\mathrm{x}=2 \mathrm{~d}$ and $\mathrm{x}=7 \mathrm{~d}$ in the experiment. The reasons for such discrepancies are not clear. For proper comparison, one needs actually to integrate along the line of sight and to account 
a)

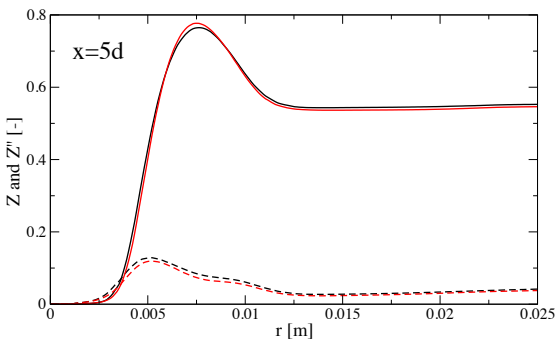

b)

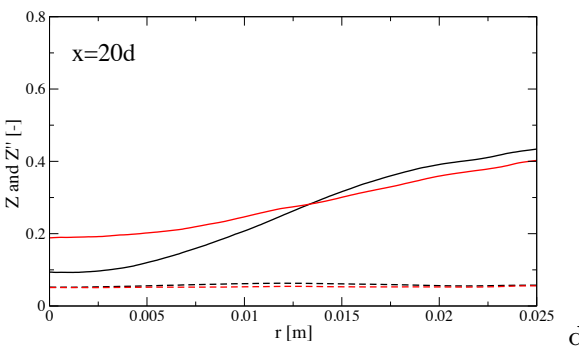

c)
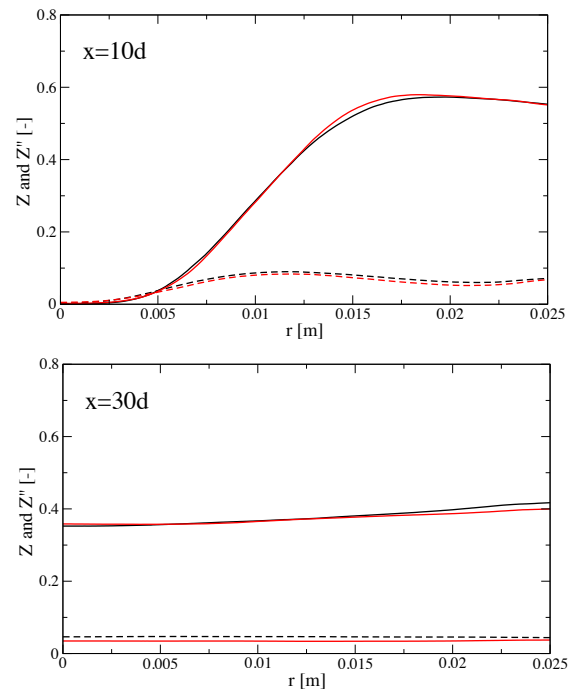

Fig. 16 Mesh convergence on case A60. Radial profiles of mean mixture fraction $Z$ (continuous lines) and its variance $Z^{\prime \prime}$ (dashed lines) at $x=5 d, x=10 d, x=20 d$ and $x=30 d$ from the injector. Black line: coarse grid, red line: fine grid.

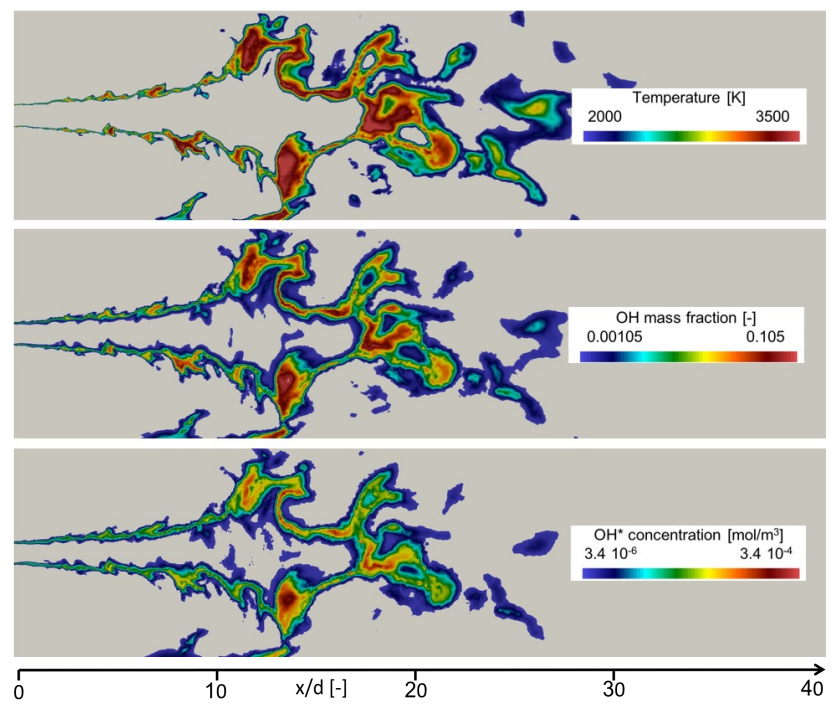

Fig. 17 Comparison between $\mathrm{OH}^{*}$ concentration, $\mathrm{OH}$ mass fraction and temperature for case A60. Instantaneous cuts of (a) temperature between $2000 \mathrm{~K}$ and $3500 \mathrm{~K}$, (b) $\mathrm{OH}$ mass fraction between 0.00105 and 0.105 and (c) $\mathrm{OH}^{*}$ emisson between $3.410^{-6}$ and $3.410^{-4} \mathrm{~mol} / \mathrm{m}^{3}$.

for absorption and re-emission during integration and the presence of the high density jet, and eventually to perform an Abel transform from the reconstructed emission from the flame. This very difficult task has not been attempted here. Finally, large-eddy simulation properly reproduces these transcritical flames, validating the solver and the methodology. The flame spreading rate at $\mathrm{x}=12 \mathrm{~d}$ for case $\mathrm{C} 60$ seems to be better captured in this work compared with the previous simulation shown in Schmitt et al. [51]. The difference between the two simulations may probably come from the use of the thickened flame model in [51], theoretically not adapted to diffusion flames. Also, an over-prediction of the flame length for case G2 was obtained in [27], certainly because of the thickened lips that were used. The flame length is now well captured is this work. 

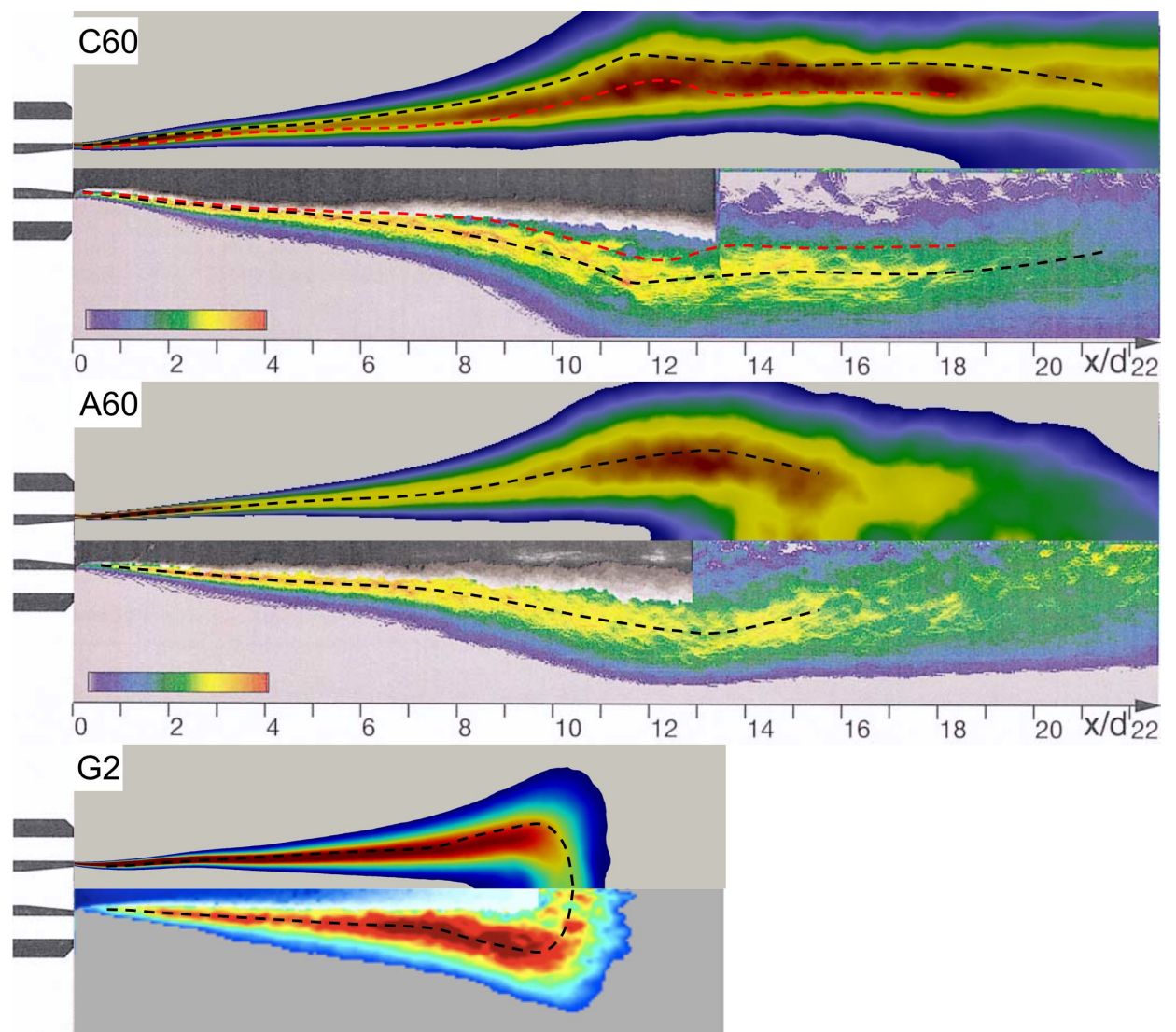

Fig. 18 Comparison with experimental visualizations. Upper half: Mean $\mathrm{OH}^{*}$ concentration from LES (between $1.1 \mathrm{e}-6$ and $1.1 \mathrm{e}-5 \mathrm{~mol} / \mathrm{m}^{3}$ for case $\mathrm{C} 60,3 \mathrm{e}-6$ and $1 \mathrm{e}-5 \mathrm{~mol} / \mathrm{m}^{3}$ for case $\mathrm{A} 60$ and $1 \mathrm{e}-6$ and $1 \mathrm{e}-5 \mathrm{~mol} / \mathrm{m}^{3}$ for case G2). Lower half: Abel transform of $\mathrm{OH}^{*}$ emission from experiments [88,82]. $d$ is the oxygen injector diameter. Dashed lines show the top (in red) and bottom (in black) experimental flame position corresponding to the position of maximum emission.

\section{Results and discussion}

5.1 Flame topology and flow dynamics

\subsubsection{Flame topology}

The interaction between the flame and the flow is illustrated for the three cases with a temperature isosurface colored with axial velocity in Fig. 19. Three zones can be distinguished. From the injector and up to $x \approx 8 \mathrm{~d}$ ( $d$ is the oxygen injector diameter), the flame is characterized by a limited spreading angle with intense and small sized turbulent structures generated by the strong shear between the two jets. Between $x \approx 8 \mathrm{~d}$ and $x \approx 12 \mathrm{~d}$, these small intense structures decrease in intensity and increase in size, and transition towards larger scale dynamics occurs, leading to a larger jet opening. Finally for $x>12$ d, the jets fully expand, large scales dominate the flow and turbulence decreases. Such a flame topology has not been observed so far with unconfined flames $[53,54]$. The sudden expansion of the flame together with the change in flow dynamics around 10d is therefore expected to be the consequence of the confinement of the jets by the walls.

\subsubsection{Flame expansion}

Longitudinal slices of instantaneous and mean axial velocity are shown in Fig. 20. The annular jet interacts with the walls and two recirculation zones are established. The first one is located between the annular jet and the walls. The second one, highly unsteady and dynamic, lies between the high velocity annular stream and the high density inner jet for cases A60 and C60, while a central recirculation region is created for case G2, explaining the sudden termination of the flame 


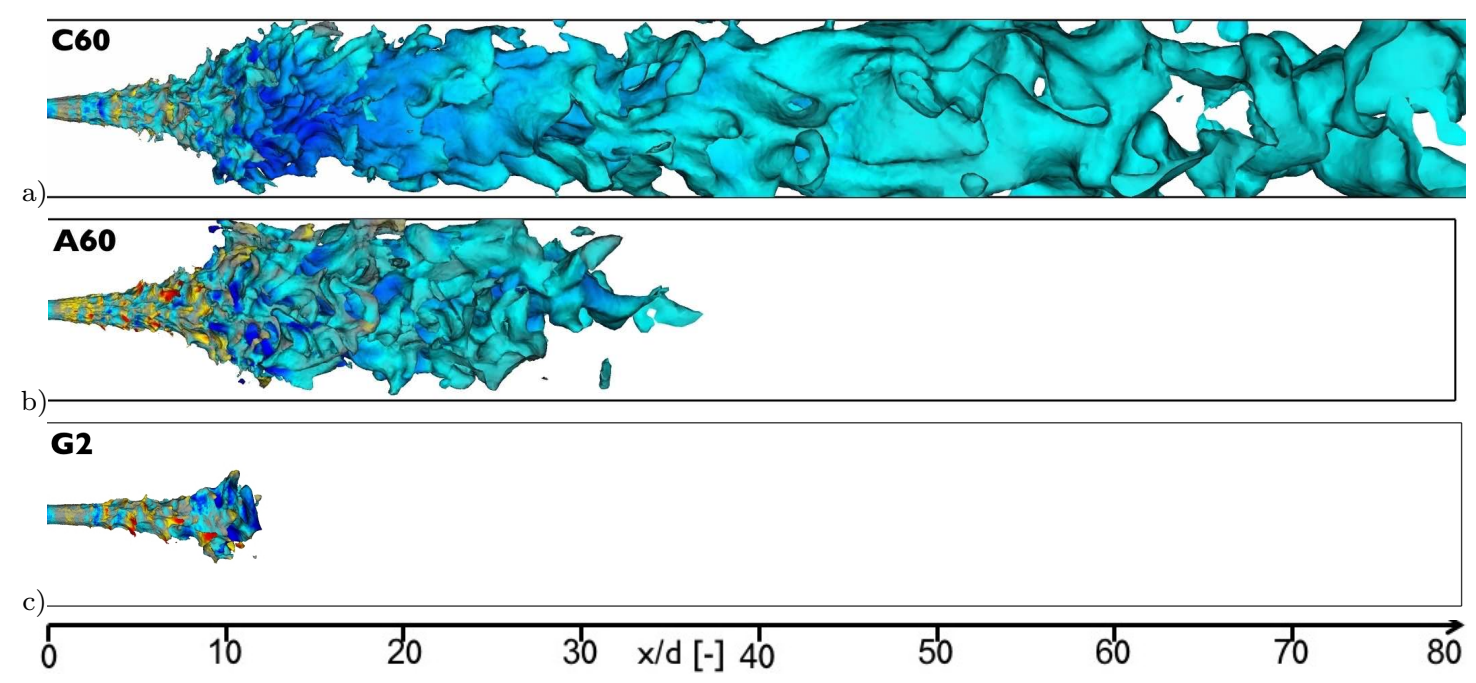

Fig. 19 Flame topology. Instantaneous iso-contour of temperature $(2000 \mathrm{~K})$ colored by the axial velocity $u_{x}$ (red : $u_{x}>50 \mathrm{~m} \mathrm{~s}^{-1}$; blue: $u_{x}<-10 \mathrm{~m} \mathrm{~s}^{-1}$ ). (a) Case C60, (b) case A60 and (c) case G2.

[27]. The size and intensity of the recirculation region seem to increase with the momentum flux ratio.
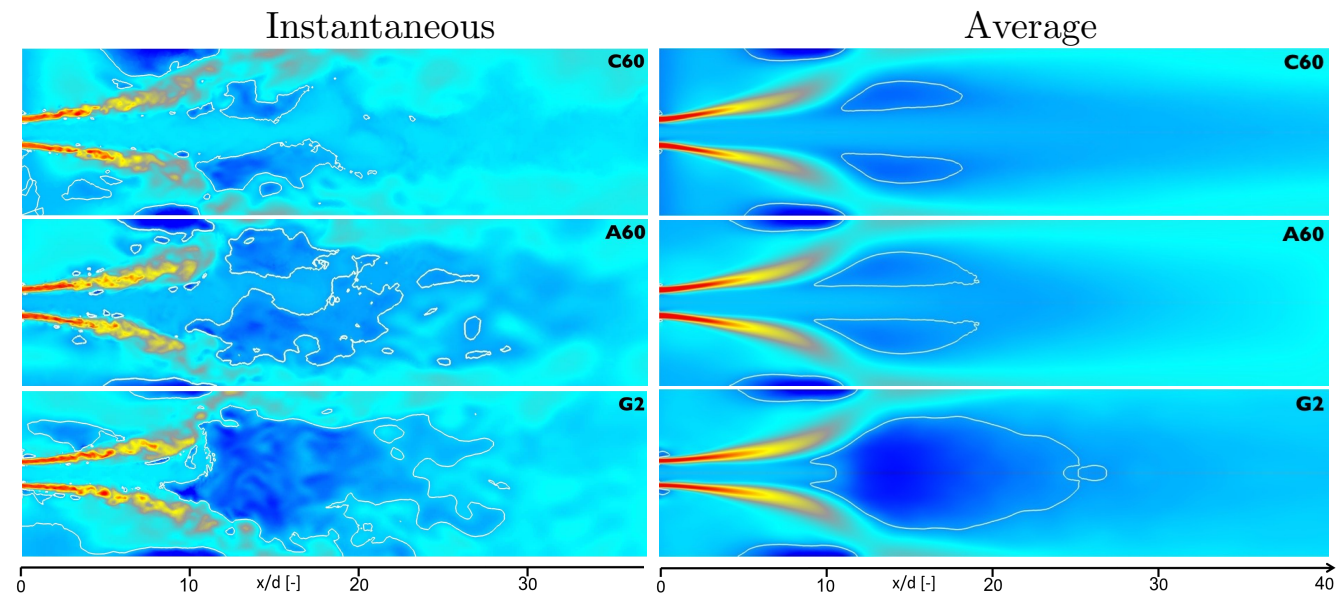

Fig. 20 Flame dynamics. Longitudinal slices of instantaneous (left) and mean (right) axial velocity (red : $u_{x}>100$ $\mathrm{m} \mathrm{s}^{-1}$; blue: $u_{x}<20 \mathrm{~m} \mathrm{~s}^{-1}$; Minimum and maximum of the colormaps are adapted to each case). White isolines show axial velocity equal to zero.

This is illustrated in Fig. 21a where the mean axial velocity is shown for the three simulations. A minimum of axial velocity is present around $22 \mathrm{~d}$ for case C60, 19d for case A60 and 13d for case G2. Its value decreases as $\mathrm{J}$ is increased, and become strongly negative for case G2 leading to the sudden drop of oxygen mass fraction observed in Fig. 21b. As a consequence, the flame length, defined in this work as the distance from the injector at which the oxygen mass fraction is lower than 0.1 , is around $12 \mathrm{~d}$ for case G2, 30d for case A60 and 62d for case C60 (Fig. 21b).

\subsection{Comparison between cases A60 and C60}

Cases A60 and C60 are compared in the following to characterize the role of fuel injection velocity (a comparison with case G2 will be less immediate since it is based on a different fuel and oxygen mass flow rate). Longitudinal slices of instantaneous density are shown in Fig. 22. The two inner jets present similar destabilization dynamics. Therefore, the axial distance at which density has 
a)

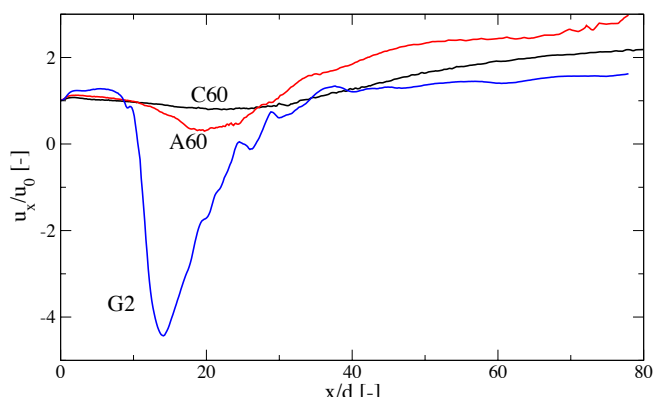

b)

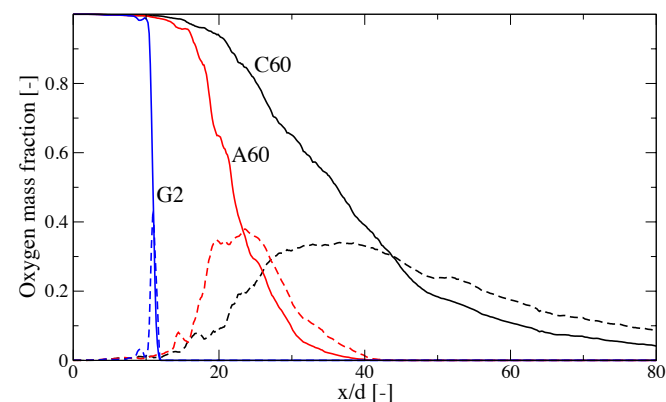

Fig. 21 Flame length. Centerline profiles of (a) mean axial velocity and (b) mean (continuous) and rms (dashed) oxygen mass fraction.

decreased to $50 \%$ of its injection value is the same for both cases $(6.5 \mathrm{~d})$. The inner jet destabilization seems to be only weakly affected by the change in annular stream velocity. This is also deduced by looking at the oxygen intact core length (distance at which the oxygen mass fraction is lower than 0.99), equal to 11.3d for case A60 and 13.5d for case C60 (Fig. 21b). However, stronger differences may be observed further downstream. There is a much longer penetration of oxygen in the domain in case C60 characterized by strong oxygen intermittency in the second part of the domain $(x>30 \mathrm{~d})$. The formation of large scale pockets of oxygen detached from the inner jet end surrounded by the flame is observed only in case C60 (Fig. 22).

A comparison of the axial distribution of heat release is plotted in Fig. 23. Note that the power of the flame is the same for the two cases considered since hydrogen is in excess. The heat release rate is 1.5 times higher in the initial mixing layer for case A60 than for case C60, proportional with the increase of inlet velocity between the two cases. There is a first maximum of heat release after the inner jet destabilization $(x \approx 4 \mathrm{~d})$. A much stronger increase of heat release, 2 times larger for case A60 than $\mathrm{C} 60$, is measured further downstream $(x=10 \mathrm{~d})$, that corresponds to the region of recirculation induced by the confinement that locally enhances mixing and combustion.

Finally, the main difference between the two cases is a higher heat release rate (and thus an higher oxygen consumption rate) for case A60 because of the stronger shear in the reactive layer. On the contrary, as shown in Fig. 24, the inner jet destabilization seems only weakly affected by the difference of outer velocity between the two cases. The initial decrease of the inner high density jet is similar between the two cases, and density fluctuations are of the same level. Moreover, the jets opening occurs at the same axial position. Because of turbulent diffusion, shear and mixing decrease downstream of the injector. Combustion is then less effective. Since oxygen consumption rate is weaker in case C60 compared to case A60, part of the oxygen is still available in case C60 as A60 flame is terminating. Since the efficiency of the combustion decreases with the distance from the injector, the distance required to burn the remaining oxygen is very long and notably increase the flame length in case C60. As a consequence, it seems that the difference between the two cases is not linked to a distinct behavior of each inner jets, but could be associated with an increased combustion efficiency for case A60 because of the effect of a higher turbulent mixing combined with a progressive decrease of combustion efficiency further downstream.

\section{Conclusions}

This article presents the large-eddy simulation of three reactive coaxial jets corresponding to cases A60, C60 and G2 experimentally studied in the Mascotte test rig [4,8]. These experiments operate at supercritical pressure for reactants, whereas oxygen is transcritical at injection. Fluids show strong departure from a perfect gas behavior and a real-gas formulation is needed to model the fluid state. The AVBP solver was adapted accordingly, using a cubic equation of state. A good representation of the flame shapes is obtained for all cases validating the developed approach. The flow is characterized by small and intense vortices in the near injector region. Further downstream, confinement influences the flow by enhancing mixing and modifying turbulent structures as the outer jets impinge the walls. By comparing cases A60 and C60, which only differ for the annular 


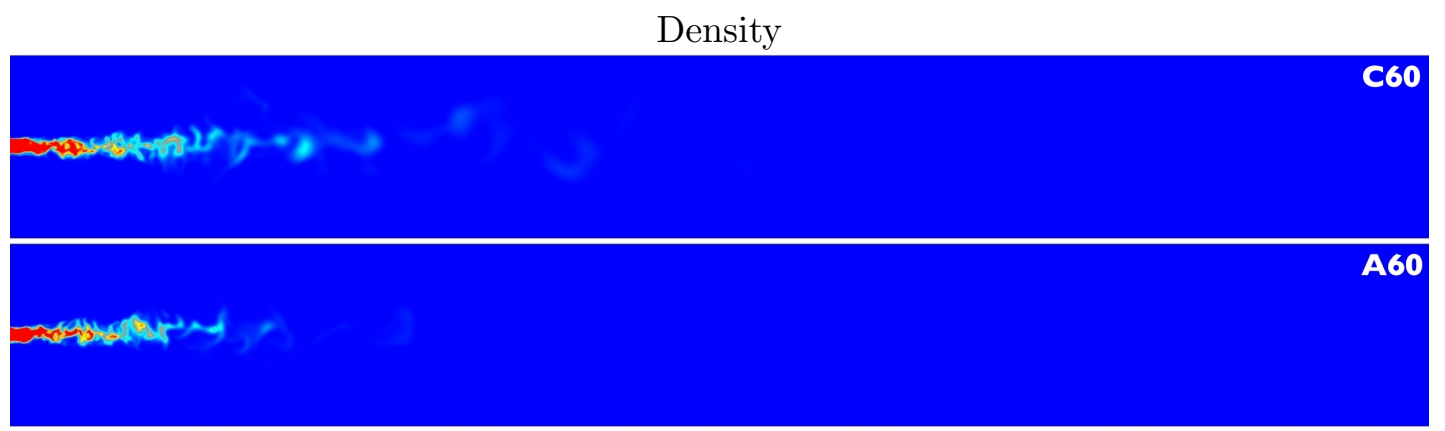

Oxygen mass fraction
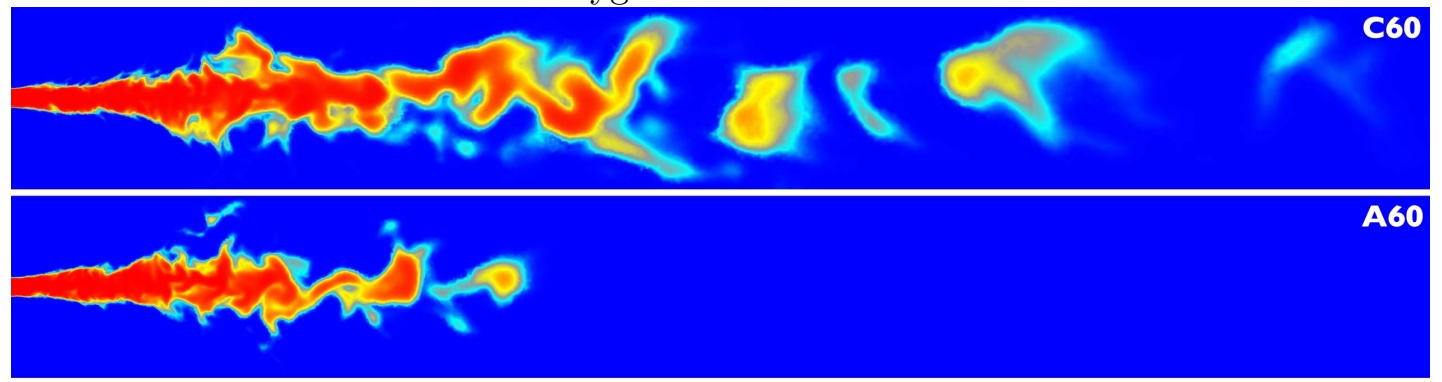

Temperature

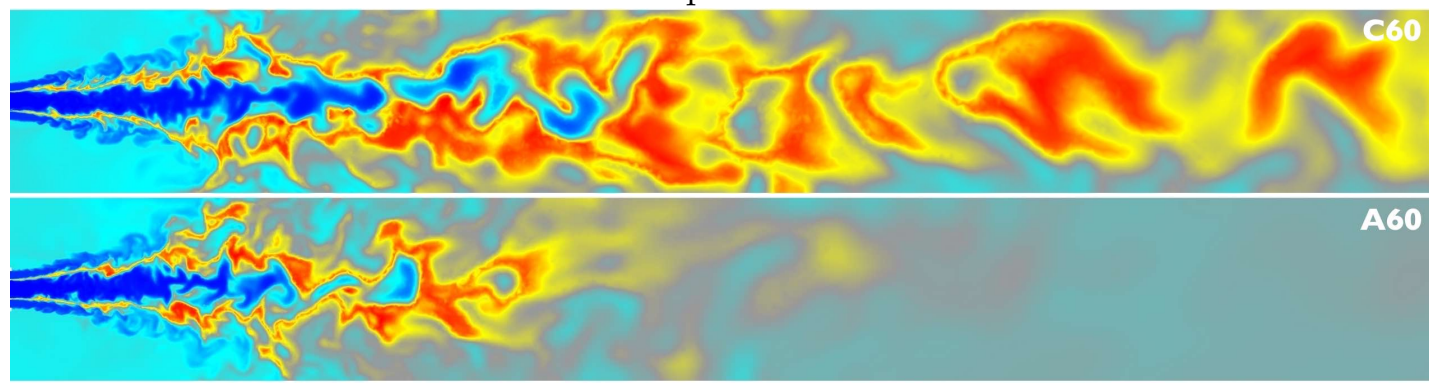

Axial velocity

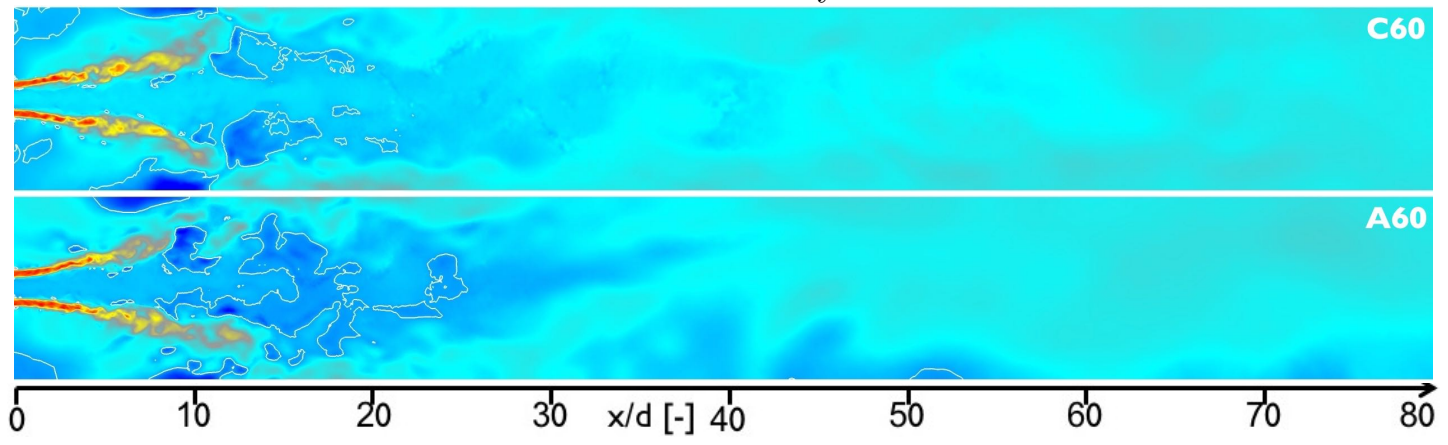

Fig. 22 Comparison between cases A60 and C60. Longitudinal slices of instantaneous density, oxygen mass fraction, temperature and axial velocity.

injection velocity and mass flow rate, a strong impact of the momentum flux ratio $J$ on the flame length is observed. It is shown that this behavior is not primarily associated to a modified destabilization of the inner high density jet. It is actually due to a combined effect of the lowered initial shear, which reduces the initial consumption rate, and the drop of combustion efficiency downstream of the injector. The strategy proposed here can accurately represent high pressure transcritical flame in rocket engines and has been applied for the simulation of complex cases [56]. However, combustion modeling could be improved to account for chemistry effect $[43,72,36,30,73$, $90,34]$, that may become influential in some extreme cases. Current works focus on combustion instability prediction using LES $[91,56]$ and extension of thermodynamics to handle two-phase and transcritical flows within the same framework. This last point is currently an active field of research $[6,92-94]$. 
a)

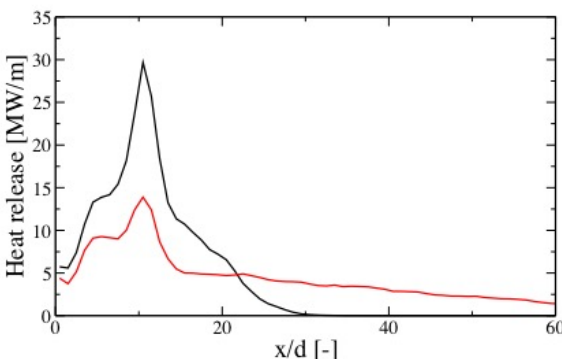

b)

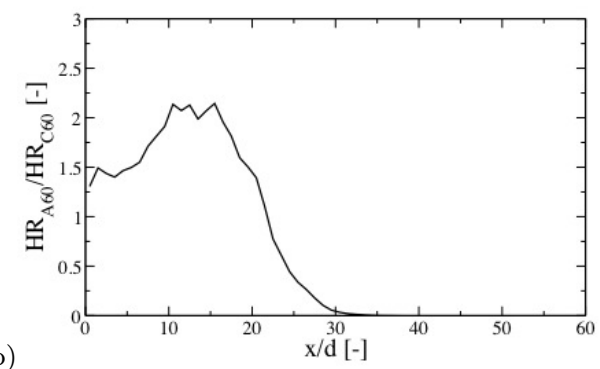

Fig. 23 Comparison between cases $A 60$ and C60. (a) Mean heat release rate as a function of the axial distance from injector for cases A60 (black line) and C60 (red line). (b) Ratio between the mean heat release of case A60 and the one of case $\mathrm{C} 60$.

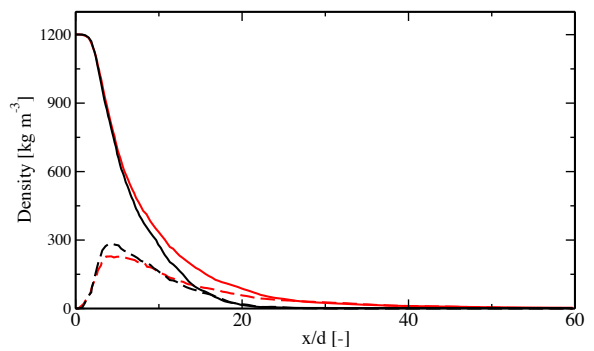

Fig. 24 Comparison between cases A60 and C60. Centerline mean and rms density (continuous lines and dashed lines, respectively) as as function of the axial distance for cases A60 (black line) and C60 (red line).

Acknowledgements Support provided by ArianeGroup, the prime contractor of the Ariane launcher cryogenic propulsion system and CNES, the French National Space Agency, is gratefully acknowledged. The author also greatly acknowledge Robin Nez from EM2C, Bénédicte Cuenot and Gabriel Staffelbach from Cerfacs and Laurent Selle from IMFT for their contributions in the AVBP-RG project. This work was granted access to the HPC resources of IDRIS and CINES made available by GENCI (Grand Equipement National de Calcul Intensif) under the allocation A0042B06176. A part of this work was performed using HPC resources from the mesocentre computing center of Ecole CentraleSupélec and Ecole Normale Supérieure Paris-Saclay supported by CNRS and Région Ile-de-France.

\section{Compliance with Ethical Standards}

Conflict of Interest The author declares that he has no conflict of interest.

\section{A Jacobian matrices for TTG schemes}

The general expression for the Two-Step Taylor Galerkin schemes [76] used in AVBP are given by :

$$
\begin{aligned}
\overrightarrow{\vec{w}}^{n} & =\vec{w}^{n}-\alpha \Delta t \vec{\nabla} \cdot \overline{\bar{F}}^{n}+\beta \Delta t^{2} \vec{\nabla} \cdot\left(\overline{\overline{\overline{\mathcal{A}}}}\left(\vec{\nabla}^{n} \cdot \overline{\bar{F}}^{n}\right)\right) \\
\vec{w}^{n+1} & =\vec{w}^{n}-\Delta t \vec{\nabla} \cdot\left(\theta_{1} \overline{\overline{\tilde{F}}}^{n}+\theta_{2} \overline{\bar{F}}^{n}\right)+\gamma \Delta t^{2}\left(\epsilon_{1} \nabla \cdot\left(\overline{\overline{\overline{\mathcal{A}}}}\left(\vec{\nabla}_{\bar{\nabla}} \overline{\bar{F}}^{n}\right)\right)+\epsilon_{2} \nabla \cdot\left(\overline{\overline{\overline{\mathcal{A}}}}\left(\vec{\nabla}^{n} \cdot \overline{\overline{\tilde{F}}}^{n}\right)\right)\right)
\end{aligned}
$$

where $\sim$ corresponds to values computed at the intermediate step. The coefficients $\alpha, \beta, \theta_{1}, \theta_{2}, \epsilon_{1}$ et $\epsilon_{2}$ are set to $0.49,1 / 6,0,1,0.01$ et 0 for TTGC. The tensor $\overline{\overline{\mathcal{A}}}=(\overline{\bar{A}}, \overline{\bar{B}}, \overline{\bar{C}})$ is the vector of the jacobian of the convective flux tensor, where $\overline{\bar{A}}, \overline{\bar{B}}$ et $\overline{\bar{C}}$ are the jacobian matrixes of the non-viscous fluxes given by :

$$
\overline{\bar{A}}(\vec{w})=\frac{\partial \vec{f}}{\partial \vec{w}} \quad \overline{\bar{B}}(\vec{w})=\frac{\partial \vec{g}}{\partial \vec{w}} \quad \overline{\bar{C}}(\vec{w})=\frac{\partial \vec{h}}{\partial \vec{w}}
$$

with $\vec{w}=\left(\rho u_{1}, \rho u_{2}, \rho u_{3}, \rho E, \rho Y_{k}\right)^{T}$ and $\overline{\bar{F}}=(\vec{f}, \vec{g}, \vec{h})$ the inviscid flux matrix:

$$
\vec{f}=\left(\begin{array}{c}
\rho u_{1}^{2}+P \\
\rho u_{1} u_{2} \\
\rho u_{1} u_{3} \\
\rho u_{1} H \\
\rho_{k} u_{1}
\end{array}\right) \vec{g}=\left(\begin{array}{c}
\rho u_{1} u_{2} \\
\rho u_{2}^{2}+P \\
\rho u_{2} u_{3} \\
\rho u_{2} H \\
\rho_{k} u_{2}
\end{array}\right) \vec{h}=\left(\begin{array}{c}
\rho u_{1} u_{3} \\
\rho u_{2} u_{3} \\
\rho u_{3}^{2}+P \\
\rho u_{3} H \\
\rho_{k} u_{3}
\end{array}\right)
$$


where $H=E+p / \rho$ is the sensible total enthalpy and

$$
\begin{gathered}
\overline{\bar{A}}=\left(\begin{array}{ccccc}
u_{1}(2-\Lambda) & -u_{2} \Lambda & -u_{3} \Lambda & \Lambda & \Gamma_{k}+\Lambda e_{c}-u_{1}^{2} \\
u_{2} & u_{1} & 0 & 0 & -u_{1} u_{2} \\
u_{3} & 0 & u_{1} & 0 & -u_{1} u_{3} \\
H-\Lambda u_{1}^{2} & -u_{1} u_{2} \Lambda & -u_{1} u_{3} \Lambda & u_{1}(1+\Lambda) & u_{1}\left(-H+\Gamma_{k}+\Lambda e_{c}\right) \\
Y_{k} & 0 & 0 & 0 & u_{1}\left(1-Y_{k}\right)
\end{array}\right) \\
\overline{\bar{B}}=\left(\begin{array}{ccccc}
u_{2} & u_{1} & 0 & 0 & -u_{1} u_{2} \\
-u_{1} \Lambda & u_{2}(2-\Lambda) & -u_{3} \Lambda & \Lambda & \Gamma_{k}+\Lambda e_{c}-u_{2}^{2} \\
0 & u_{3} & u_{2} & 0 & -u_{2} u_{3} \\
-u_{1} u_{2} \Lambda & H-\Lambda u_{2}^{2} & -u_{2} u_{3} \Lambda & u_{2}(1+\Lambda) & u_{2}\left(-H+\Gamma_{k}+\Lambda e_{c}\right) \\
0 & Y_{k} & 0 & 0 & u_{2}\left(1-Y_{k}\right)
\end{array}\right) \\
\overline{\bar{C}}=\left(\begin{array}{ccccc}
u_{3} & 0 & u_{1} & 0 & -u_{1} u_{3} \\
0 & u_{3} & u_{2} & 0 & -u_{2} u_{3} \\
-u_{1} \Lambda & -u_{2} \Lambda & u_{3}(2-\Lambda) & \Lambda & \Gamma_{k}+\Lambda e_{c}-u_{3}^{2} \\
-u_{1} u_{3} \Lambda & -u_{2} u_{3} \Lambda & H-\Lambda u_{3}^{2} & u_{3}(1+\Lambda) & u_{3}\left(-H+\Gamma_{k}+\Lambda e_{c}\right) \\
0 & 0 & Y_{k} & 0 & u_{3}\left(1-Y_{k}\right)
\end{array}\right)
\end{gathered}
$$

with the coefficients $\Omega_{k}$ and $\Lambda$ given by:

$$
\begin{array}{r}
\Gamma_{k}=\frac{C_{p} v_{k}}{C_{v} \beta}-\Lambda h_{k} \\
\Lambda=\frac{\alpha}{\rho \beta C_{v}}
\end{array}
$$

In Eq. 33 and $34, C_{p}$ and $C_{v}$ are the heat capacities at constant pressure and volume, $\alpha$ is the thermal expansion coefficient and $\beta$ is the isothermal compressibility coefficient. Partial-mass volume and enthalpy are $v_{k}$ and $h_{k}$, respectively and $e_{c}=1 / 2 \sum_{i=1}^{3} u_{i}^{2}$ is the kinetic energy.

\section{References}

1. B.E. Poling, J.M. Prausnitz, J.P. O'Connel, The properties of gases and liquids, 5th edn. (McGraw-Hill, 2001)

2. M. Oschwald, J.J. Smith, R. Branam, J. Hussong, A. Shick, B. Chehroudi, D. Talley, Combustion Science and Technology 178, 49 (2006)

3. C. Segal, S. Polikhov, Physics of Fluids 20, 052101 (2008)

4. M. Habiballah, M. Orain, F. Grisch, L. Vingert, P. Gicquel, Combustion Science and Technology 178(1), 101 (2006)

5. P. Gaillard, V. Giovangigli, L. Matuszewski, Combustion Theory and Modelling 20(3), 486 (2016)

6. J. Matheis, S. Hickel, International Journal of Multiphase Flow 99, 294 (2018)

7. C. Traxinger, J. Zips, M. Pfitzner, Journal of Propulsion and Power 35(4), 675 (2019)

8. S. Candel, M. Juniper, G. Single, P. Scouflaire, C. Rolon, Combustion Science and Technology 178, 161 (2006)

9. J. Bellan, Combustion Science and Technology 178(1), 253 (2006)

10. J. Oefelein, Proceedings of the Combustion Institute 30(2), 2929 (2005)

11. L. Pons, N. Darabiha, S. Candel, Combustion and Flame 152(1-2), 218 (2008)

12. G. Ribert, N. Zong, V. Yang, L. Pons, N. Darabiha, S. Candel, Combustion and Flame 154(3), 319 (2008)

13. V. Giovangigli, L. Matuszewski, F. Dupoirieux, Combustion Theory and Modelling 15(2), 141 (2011)

14. V. Giovangigli, L. Matuszewski, Combustion and Flame 159(9), 2829 (2012)

15. D.T. Banuti, P.C. Ma, J.P. Hickey, M. Ihme, Combustion and Flame 196, 364 (2018)

16. J. Bellan, Progress in Energy and Combustion Science 26, 329 (2000)

17. L. Selle, N. Okong'o, J. Bellan, K. Harstad, Journal of Fluid Mechanics 593, 57 (2007)

18. E. Taşkinoğlu, J. Bellan, Journal of Fluid Mechanics 679, 156 (2011)

19. J. Bellan, Combustion and Flame 176, 245 (2017)

20. P.E. Lapenna, F. Creta, The Journal of Supercritical Fluids 128, 263 (2017)

21. F. Demoulin, S. Zurbach, A. Mura, Journal of propulsion and power 25(2) (2009)

22. M. Poschner, M. Pfitzner, in Proceedings of the European Combustion Meeting 2009 (2010)

23. N. Zong, V. Yang, Combustion Science and Technology 178(1), 193 (2006)

24. J. Oefelein, Combustion Science and Technology 178(1), 229 (2006)

25. M. Masquelet, S. Menon, Y. Jin, R. Friedrich, Aerospace Science and Technology 13(8), 466 (2009)

26. H. Terashima, S. Kawai, N. Yamanishi, AIAA journal 49(12), 2658 (2011)

27. T. Schmitt, Y. Méry, M. Boileau, S. Candel, Proceedings of the Combustion Institute 33(1), 1383 (2011)

28. T. Schmitt, J. Rodriguez, I. Leyva, S. Candel, Physics of Fluids 24(5), 055104 (2012)

29. X. Petit, G. Ribert, G. Lartigue, P. Domingo, The Journal of Supercritical Fluids 84, 61 (2013)

30. X. Petit, G. Ribert, P. Domingo, The Journal of Supercritical Fluids 101, 1 (2015)

31. S. Kawai, H. Terashima, H. Negishi, Journal of Computational Physics 300, 116 (2015)

32. J. Matheis, H. Müller, C. Lenz, M. Pfitzner, S. Hickel, The Journal of Supercritical Fluids 107, 422 (2016)

33. P.C. Ma, Y. Lv, M. Ihme, Journal of Computational Physics 340, 330 (2017) 
34. P.C. Ma, D. Banuti, J.P. Hickey, M. Ihme, in 55th AIAA Aerospace Sciences Meeting (2017), p. 0143

35. N. Zong, G. Ribert, V. Yang, AIAA paper 946, 2008 (2008)

36. G. Lacaze, J.C. Oefelein, Combustion and Flame 159(6), 2087 (2012)

37. J. Zips, H. Müller, M. Pfitzner, Flow, Turbulence and Combustion 101(3), 821 (2018)

38. S. Karni, Journal of Computational Physics 112, 31 (1994)

39. R. Abgrall, Journal of Computational Physics 125, 150 (1996)

40. G. Lacaze, T. Schmitt, A. Ruiz, J. Oefelein, Computers and Fluids 181, 35 (2019)

41. H. Meng, V. Yang, Journal of Computational Physics 189, 277 (2003)

42. H. Müller, M. Pfitzner, J. Matheis, S. Hickel, Journal of Propulsion and Power 32(1), 46 (2015)

43. H. Müller, M. Pfitzner, in 6th European Conference for Aeronautics and Space Sciences (EUCASS), Krakau, Poland (2015)

44. H. Müller, C.A. Niedermeier, J. Matheis, M. Pfitzner, S. Hickel, Physics of fluids 28(1), 015102 (2016)

45. T. Schmitt, L. Selle, A. Ruiz, B. Cuenot, AIAA JOURNAL 48(9) (2010)

46. C. Pantano, R. Saurel, T. Schmitt, Journal of Computational Physics 335, 780 (2017)

47. N. Zong, V. Yang, Proceedings of the Combustion Institute 31(2), 2309 (2007)

48. A.M. Ruiz, G. Lacaze, J.C. Oefelein, R. Mari, B. Cuenot, L. Selle, T. Poinsot, AIAA Journal 54(5), 1445 (2015)

49. C. Laurent, L. Esclapez, D. Maestro, G. Staffelbach, B. Cuenot, L. Selle, T. Schmitt, F. Duchaine, T. Poinsot, Proceedings of the Combustion Institute (2018)

50. X. Wang, H. Huo, U. Unnikrishnan, V. Yang, Combustion and Flame 195, 203 (2018)

51. T. Schmitt, L. Selle, B. Cuenot, T. Poinsot, Comptes Rendus Mécanique 337(6-7), 528 (2009)

52. S. Matsuyama, J. Shinjo, S. Ogawa, Y. Mizobuchi, in 48th AIAA Aerospace Sciences Meeting, Orlando, Florida (2010)

53. L. Hakim, A. Ruiz, T. Schmitt, M. Boileau, G. Staffelbach, S. Ducruix, B. Cuenot, S. Candel, Proceedings of the Combustion Institute 35(2), 1461 (2015)

54. L. Hakim, T. Schmitt, S. Ducruix, S. Candel, Combustion and Flame 162(10), 3482 (2015)

55. T. Schmitt, A. Coussement, S. Ducruix, S. Candel, in Proceedings of Space Propulsion (2016)

56. T. Schmitt, G. Staffelbach, S. Ducruix, S. Gröning, J. Hardi, M. Oschwald, in 7TH European Conference for Aeronautics and Aerospace Sciences (EUCASS) (2017)

57. B. Chehroudi, D. Talley, E. Coy, Physics of Fluids 14(2), 850 (2002)

58. T. Poinsot, D. Veynante, Theoretical and numerical combustion (R.T. Edwards, 2nd edition., 2005)

59. L.L. Lee, K.E. Starling, T.H. Chung, M. Ajlan, Industrial and Chemical Engineering Research 27, 671 (1988)

60. A.J. Juanós, W.A. Sirignano, Combustion and Flame 181, 54 (2017)

61. F. Nicoud, F. Ducros, Flow, Turbulence and Combustion 62(3), 183 (1999). JX

62. G. Borghesi, J. Bellan, Physics of Fluids 27(035117) (2015)

63. G. Soave, Chemical Engineering Science 27, 1197 (1977)

64. E. Lemmon, M. McLinden, D. Friend, NIST Chemistry WebBook, NIST Standard Reference Database Number 69 (National Institute of Standards and Technology, Gaithersburg MD, 20899, 2009), chap. Thermophysical Properties of Fluid Systems

65. W. Mayer, H. Tamura, Journal of Propulsion and Power 12(6), 1137 (1996)

66. B. Ivancic, W. Mayer, Journal of Propulsion and Power 18(2), 247 (2002)

67. A. Ruiz, B. Cuenot, L. Selle, T. Poinsot, in 47th AIAA/ASME/SAE/ASEE Joint Propulsion Conference E Exhibit (2011), p. 6121

68. R. Mari, B. Cuenot, F. Duchaine, L. Selle, in Proceedings of the Summer Program (Citeseer, 2012), p. 439

69. D. Veynante, L. Vervisch, Progress in Energy and Combustion Science 28, 193 (2002)

70. P. Domingo, L. Vervisch, D. Veynante, Combustion and Flame 152(3), 415 (2008)

71. C. Pera, O. Colin, S. Jay, Oil \& Gas Science and Technology-Revue de l'IFP 64(3), 243 (2009)

72. N. Zong, V. Yang, Proceedings of the Combustion Institute 31(2), 2309 (2007)

73. H. Huo, V. Yang, Journal of Propulsion and Power 33(5), 1272 (2017)

74. V. Moureau, G. Lartigue, Y. Sommerer, C. Angelberger, O. Colin, T. Poinsot, Journal of Computational Physics 202(2), 710 (2005)

75. T. Schönfeld, T. Poinsot, in Annual Research Briefs (Center for Turbulence Research, NASA Ames/Stanford Univ., 1999), pp. 73-84

76. O. Colin, M. Rudgyard, Journal of Computational Physics 162(2), 338 (2000)

77. N. Okong'o, J. Bellan, Journal of Computational Physics 176, 330 (2002)

78. J. Mathew, R. Lechner, H. Foysi, J. Sesterhenn, R. Friedrich, Physics of fluids 15(8), 2279 (2003)

79. S. Stolz, N.A. Adams, Physics of Fluids 11(7), 1699 (1999)

80. L. Vingert, M. Habiballah, P. Vuillermoz, S. Zurbach, in 51st International Astronautical Congress, Rio de Janeiro, Brazil (2000)

81. M. Juniper, Structure et stabilisation des flammes cryotechniques. Ph.D. thesis, Ecole Centrale de Paris (2001)

82. G. Singla, P. Scouflaire, C. Rolon, S. Candel, Proceedings of the Combustion Institute 30(2), 2921 (2005)

83. P. Schmitt, T.J. Poinsot, B. Schuermans, K. Geigle, Journal of Fluid Mechanics 570, 17 (2007)

84. F. Jaegle, O. Cabrit, S. Mendez, T. Poinsot, Flow, turbulence and combustion 85(2), 245 (2010)

85. T. Poinsot, S. Lele, Journal of Computational Physics 101(1), 104 (1992)

86. R. Kraichnan, Physics of Fluids 13, $22(1970)$

87. A. Smirnov, S. Shi, I. Celik, Trans. ASME. Journal of Fluids Engineering 123, 359 (2001)

88. M. Juniper, A. Tripathi, P. Scouflaire, J. Rolon, S. Candel, Proceedings of the Combustion Institute 28(1), $1103(2000)$

89. T. Fiala, T. Sattelmayer, in 5th European Conference for Aeronautics and Space Sciences (EUCASS), Munich, July (2013), pp. 1-5

90. N. Zong, V. Yang, Physics of Fluids 20, 056103 (2008)

91. A. Urbano, L. Selle, G. Staffelbach, B. Cuenot, T. Schmitt, S. Ducruix, S. Candel, Combustion and Flame 169, $129(2016)$

92. G. Castiglioni, J. Bellan, Journal of Fluid Mechanics 843, 536 (2018)

93. M. Pelletier, S. Thomas, S. Ducruix, in ICLASS 2018 (2018)

94. D. Nayigizente, S. Thomas, S. Ducruix, in ICLASS 2018 (2018) 